\title{
MODELLING ELECTRICITY FUTURES BY AMBIT FIELDS
}

\author{
OLE E. BARNDORFF-NIELSEN, ${ }^{*}$ Aarhus University \\ FRED ESPEN BENTH, ${ }^{* *}$ University of Oslo \\ ALMUT E. D. VERAART, ${ }^{* * *}$ Imperial College London
}

\begin{abstract}
In this paper we propose a new modelling framework for electricity futures markets based on so-called ambit fields. The new model can capture many of the stylised facts observed in electricity futures and is highly analytically tractable. We discuss martingale conditions, option pricing, and change of measure within the new model class. Also, we study the corresponding model for the spot price, which is implied by the new futures model, and show that, under certain regularity conditions, the implied spot price can be represented in law as a volatility modulated Volterra process.
\end{abstract}

Keywords: Electricity market; futures price; random field; ambit field; Lévy base; Samuelson effect; stochastic volatility

2010 Mathematics Subject Classification: Primary 91G99

Secondary 60G51; 60G57; 60G60

\section{Introduction}

Over the past two decades, electricity markets have been liberalised in many areas in the world. The typical electricity market, such as, for instance, the Nordic Nord Pool market or the European Energy Exchange (EEX) market, organises trade in spot, forward/futures contracts and European options on these. Although these assets are parallel to other markets, such as traditional commodities or stock markets, electricity has its own distinctive features calling for new and more sophisticated stochastic models for derivative pricing as well as for risk management purposes; see [22].

Recent research has particularly focused on modelling electricity spot (or day-ahead) prices; see, e.g. [4], [5], [30], [41], [55], and [67]. Since electricity cannot be stored directly, except via reservoirs for hydro-generated power or large and expensive batteries, the supply of power is very inelastic, and spot prices may rise by several magnitudes when demand increases, due to temperature drops, say. Since spot prices are determined by supply and demand, some form of mean reversion or stationarity can be observed. The spot prices have clear deterministic patterns over the year, week, and intra-day. The literature has focused on stochastic models for the spot price dynamics, which take some of the various stylised facts into account. Recently, a general, yet analytically tractable class of models has been proposed in [16] based on volatility modulated Volterra processes and the subclass of Lévy semistationary processes.

\footnotetext{
Received 27 September 2011; revision received 3 April 2013.

* Postal address: Thiele Center, Department of Mathematics, and CREATES, Aarhus University, Ny Munkegade 118, DK-8000 Aarhus C, Denmark. Email address: oebn@imf.au.dk

** Postal address: Centre of Mathematics for Applications, University of Oslo, PO Box 1053, Blindern, N-0316 Oslo, Norway. Email address: fredb@math.uio.no

*** Postal address: Department of Mathematics, Imperial College London, 180 Queen's Gate, London SW7 2AZ, UK. Email address: a.veraart@imperial.ac.uk
} 
One of the fundamental problems in power market modelling is to understand the formation of futures prices. Nonstorability of the spot makes the usual buy-and-hold hedging arguments break down, and the notion of convenience yield is not relevant either; see [36], [40], and [42]. There is thus a highly complex relationship between spot and forwards, which is often modelled by risk premiums; see, e.g. [35], [55], or [64] for some recent work on this aspect. A way around this would be to follow the so-called Heath-Jarrow-Morton (HJM) approach, which has been introduced in the context of modelling interest rates, see [45], and model the futures price dynamics directly (rather than modelling the spot price and deducing the futures price from the conditional expectation of the spot at delivery); see, e.g. [32]. However, many challenging problems are connected to this way of modelling futures prices. First, standard models for the futures dynamics generally depend on the current time and the time to maturity. However, power markets trade in contracts that deliver power over a delivery period, introducing a new dimension in the modelling. Hence, comprehensive futures price models should be functions of both time to and length of delivery, which calls for random field models in time and space. Furthermore, since the market trades in contracts with overlapping delivery periods, specific noarbitrage conditions must be satisfied which essentially puts restrictions on the space structure of the field. So far, the literature is sparse on modelling power futures prices applying the HJM approach, presumably due to the lack of analytical tractability and empirical knowledge of the price evolution; however, see, e.g. [19], [47], and [52].

Empirical studies, see [38], have shown that the logarithmic returns of futures prices are nonnormally distributed with clear signs of (semi-) heavy tails. Also, a principal component analysis by Koekebakker and Ollmar [54] revealed a high degree of idiosyncratic risk in power futures markets. This strongly points towards random field models which, in addition, allow for stochastic volatility. Moreover, the structure determining the interdependencies between different contracts is by far not properly understood. Some empirical studies, see [2], suggest that the correlations between contracts are decreasing with time to maturity, whereas the exact form of this decay is not known. But how to take length of delivery into account in modelling these interdependencies has been an open question. A first approach on how to tackle these problems will be presented later in this paper.

In this paper we introduce a new modelling framework for electricity futures prices, which is based on ambit fields. Ambit fields are defined as stochastic integrals with respect to an independently scattered, infinitely divisible random measure, where the integrand is given by a product of a deterministic weight function and a stochastic volatility field, and the integration is carried out over an ambit set describing the sphere of influence for the stochastic field. Owing to their very flexible structure, ambit processes have successfully been used for modelling turbulence in physics and cell growth in biology; see [6]-[11] and [63].

In this paper we will show how the ambit concept can be used to develop a general modelling framework for electricity futures. Ambit fields form a flexible class of random field models, where one has a high degree of flexibility in modelling complex dependencies. These may be probabilistic coming from a driving random noise and the stochastic volatility, or functional from a specification of an ambit set or the deterministic weight function. Moreover, ambit fields easily incorporate leptokurtic behaviour in price increments, stochastic volatility and leverage effects, and the observed Samuelson effect in the volatility. Note that the Samuelson effect, see [58], refers to the finding that, when the time to maturity approaches 0 , the volatility of the futures increases and converges to the volatility of the underlying spot price (provided the futures price converges to the spot price).

Last but not least we will show that the ambit framework can shed some light on the connection between electricity spot and futures prices. Understanding the interdependencies between 
these two assets is crucial in many applications, e.g. in the hedging of exotic derivatives on the spot using futures. Typical examples in electricity markets are so-called flexible load/virtual power plant (VPP) contracts, which are particular kinds of swing option; see, e.g. [44].

The outline for the remaining part of the paper is as follows. In Section 2 we give a short overview of the traditional models within the HJM class used for electricity futures. In Section 3 we introduce the new modelling framework for electricity futures markets together with its key properties. The model will be specified as an arithmetic model. Examples of relevant model specifications are discussed in detail. Next we derive the implied spot price from our new model for the futures price, and we show that, under certain conditions, the implied spot price process equals in law a volatility modulated Volterra process; see Section 4. In Section 5 we describe how a geometric model based on ambit fields can be defined. Next, Section 6 focuses on option pricing in our new modelling framework. In order to also obtain a visual impression of the new models for the term structure of futures prices, we present a simulation algorithm for ambit fields in Section 7 and highlight the main theoretical properties of the modelling framework graphically. Moreover, since we do the modelling under the risk-neutral measure, we discuss how a change of measure can be carried out to get back to the physical probability measure; see Section 8. Finally, in Section 9 we conclude the paper.

\section{A brief literature review}

Although commodity markets have very distinct features, most models for energy futures contracts are inspired by models for instantaneous forward rates in the context of the term structure of interest rates; see [54] for an overview on the similarities between electricity futures markets and interest rates. Hence, in order to get an overview on modelling concepts within the HJM framework which have been developed in the context of the term structure of interest rates, but which can also be used in the context of electricity markets, we will now review these examples from the interest rate literature. However, later we will argue that, in order to account for the particular stylised facts of power markets, there is a case for leaving these models behind and focusing instead on ambit fields as a natural class for describing energy futures markets.

Throughout the paper, we denote by $t \in \mathbb{R}$ the current time, by $T \geq 0$ the time of maturity of a given futures/forward contract, and by $x=T-t$ the corresponding time to maturity. We use $F(t, T)$ to denote the price of a futures/forward contract at time $t$ with time of maturity $T$. Likewise, we use $f$ for the futures/forward price at time $t$ with time to maturity $x=T-t$ when we work with the Musiela parameterisation, i.e. we define $f$ by $f_{t}(x)=f_{t}(T-t)=F(t, T)$.

Let us begin by looking at so-called multifactor models. Motivated by the classical HJM framework (see [45]), the dynamics of the forward rate under the risk-neutral measure can be modelled by $\mathrm{d} f_{t}(x)=\sum_{i=1}^{n} \sigma_{t}^{(i)}(x) \mathrm{d} W_{t}^{(i)}$ for $t \geq 0$ and $n \in \mathbb{N}$, where the $W^{(i)}$ are independent standard Brownian motions and the $\sigma^{(i)}(x)$ are independent positive stochastic volatility processes for $i=1, \ldots, n$. The advantage of using these multifactor models is that they are to a high degree analytically tractable. Extensions to allow for jumps in such models have also been studied in detail in the literature. However, a principal component analysis by Koekebakker and Ollmar [54] has indicated that we in fact need many factors (large $n$ ) to model electricity futures prices. Hence, it is natural to study extensions to infinite-factor models which are also called random field models.

In order to overcome the shortcomings of the multifactor models, Kennedy [50] pioneered the approach of using random field models, in some cases called stochastic string models, for modelling the term structure of interest rates. Random field models have a continuum of state variables (in our case futures prices for all maturities) and, hence, are also called infinite-factor 
models, but they are typically very parsimonious in the sense that they do not require many parameters. Note that finite-factor models can be accommodated by random field models as degenerate cases.

Kennedy [50] proposed to model the forward rate by a centred, continuous Gaussian random field plus a continuous deterministic drift. Furthermore, he specified a certain structure of the covariance function of the random field which ensured that it had independent increments in the time direction $t$ (but not necessarily in the time to maturity direction $x$ ). Such models include as special cases the classical HJM model when both the drift and the volatility functions are deterministic, and also two-parameter models, such as models based on Brownian sheets. Kennedy [50] derived suitable drift conditions which ensure the martingale properties of the corresponding discounted zero-coupon bonds.

In a later article, Kennedy [51] revisited the continuous Gaussian random field models and showed that the structure of the covariance function of such models can be specified explicitly if one assumes a Markov property. Adding an additional stationarity condition, the correlation structure of such processes is already very limited and Kennedy [51] proved that, in fact, under a strong Markov and stationarity assumption, the Gaussian field is necessarily described by just three parameters.

The Gaussian assumption was later relaxed and Goldstein [43] presented a term structure model based on non-Gaussian random fields. Such models incorporate, in particular, conditional volatility models, i.e. models which allow for more flexible (i.e. stochastic) behaviour of the (conditional) volatilities of the innovations to forward rates (in the traditional Kennedy approach such variances were just constant functions of maturity), and, hence, are particularly relevant for empirical applications. Also, Goldstein [43] pointed out that one is interested in very smooth random field models in the context of modelling the term structure of interest rates. Such a smoothness (e.g. in the time to maturity direction) can be achieved by using integrated random fields, e.g. he proposed integrating over an Ornstein-Uhlenbeck process. Goldstein [43] derived drift conditions for the absence of arbitrage for such general non-Gaussian random field models.

While such models are quite general and, hence, appealing in practice, Kimmel [53] pointed out that the models defined by Goldstein [43] are generally specified as solutions to a set of stochastic differential equations, where it is difficult to prove the existence and uniqueness of solutions. Goldstein's [43] models and many other conditional volatility random field models are in fact complex and often infinite-dimensional processes, which lack the key property of the Gaussian random field models introduced by Kennedy [50], i.e. that the individual forward rates are low-dimensional diffusion processes. The latter property is in fact important for model estimation and derivative pricing. Hence, Kimmel [53] proposed a new approach to random field models which allows for conditional volatility and which preserves the key property of the Kennedy [50] class of models: the class of latent variable term structure models. He proved that such models ensure that the forward rates and the latent variables (which are modelled as a joint diffusion) follow jointly a finite-dimensional diffusion.

A different approach to generalising the Kennedy [50] framework was proposed by Albeverio et al. [1]. They suggested replacing the Gaussian random field in the Kennedy [50] model by a (pure jump) Lévy field. Special cases of such models are, e.g. the Poisson and the gamma sheet.

Finally, another approach for modelling forward rates has been proposed by Santa-Clara and Sornette [59], who built their model on stochastic string shocks. Such models are related (and under some assumptions even a special case) of the new modelling framework we present in this paper. 


\section{Arithmetic model for the futures price}

Throughout this paper, we consider a probability space $(\Omega, \mathcal{F}, \mathbb{Q})$, where the notation $\mathbb{Q}$ is used for the probability measure since it denotes a risk-neutral probability measure. We will define our new model in such a way that forward/futures prices are martingales under a risk-neutral probability measure.

\subsection{Background on Lévy bases}

In the following, we will briefly sketch the basic traits of Lévy bases; a more detailed review can be found in [15]. Recall that a Lévy basis is an infinitely divisible, independently scattered random measure; see, e.g. [56]. Let $\mathcal{B}_{b}\left(\mathbb{R}^{d}\right)$ denote the class of bounded Borel sets in $\mathbb{R}^{d}$ for $d \in \mathbb{N}$. We call $L=\left\{L(A): A \in \mathscr{B}_{b}\left(\mathbb{R}^{d}\right)\right\}$ a Lévy basis taking values in $\mathbb{R}$ if it satisfies the following three properties.

(i) For any sequence $\left(A_{n}\right)_{n \in \mathbb{N}}$ of disjoint elements of $\mathscr{B}_{b}\left(\mathbb{R}^{d}\right)$ such that $\bigcup_{j=1}^{\infty} A_{j} \in \mathcal{B}_{b}\left(\mathbb{R}^{d}\right)$, we have $L\left(\bigcup_{j=1}^{\infty} A_{j}\right)=\sum_{j=1}^{\infty} L\left(A_{j}\right)$ almost surely (a.s.).

(ii) For any finite sequence $A_{1}, \ldots, A_{n}$ of disjoint elements of $\mathcal{B}_{b}\left(\mathbb{R}^{d}\right)$, the random variables $L\left(A_{1}\right), L\left(A_{2}\right), \ldots, L\left(A_{n}\right)$ are independent.

(iii) For any $A \in \mathcal{B}_{b}\left(\mathbb{R}^{d}\right)$, the random variable $L(A)$ is infinitely divisible.

Note that we call a Lévy basis atomless if $L(\{\boldsymbol{a}\})=0$ a.s. for every one-point set $\{\boldsymbol{a}\} \subset \mathbb{R}^{d}$. Moreover, we call a Lévy basis homogeneous if $L(A)=L(A+\{\boldsymbol{a}\})$ a.s. for any $A \in \mathscr{B}_{b}\left(\mathbb{R}^{d}\right)$ and $\boldsymbol{a} \in \mathbb{R}^{d}$.

Remark 3.1. Note that the term 'random measure' has been widely used in the literature, but can potentially cause confusion in the sense that it has been used with two different definitions. Classical probability theory defines a random measures as a locally finite kernel from the underlying probability space to $\mathbb{R}$ (or to $\mathbb{R}^{d}$ for $d \in \mathbb{N}$ ); see, e.g. [48, p. 203]. However, Lévy bases defined above are generally not random measures according to that classical definition since their realisations are in general not ordinary (signed) measures; see, e.g. [57, p. 118] for a discussion of this aspect. Hence, some authors would rather call them random noise. The 'random measures' we are dealing with in the context of Lévy bases can rather be understood as set-indexed stochastic processes.

Remark 3.2. It is well known that in the one-dimensional case, i.e. when $d=1$, the process defined by

$$
L_{t}:=L([0, t])
$$

for a homogeneous Lévy basis $L$ is a Lévy process ; see [61, Proposition 3.3]. Likewise, for any Lévy process, one can find a homogeneous Lévy basis such that (3.1) holds (in law).

In the following we will often work with the so-called cumulant function, which is defined as the distinguished logarithm of the characteristic function. Recall the definition of the distinguished logarithm; see, e.g. [31, p. 255] or [60, p. 33].

Definition 3.1. Let $g(z)$ be a complex-valued continuous function on $\mathbb{R}$ satisfying $g(0)=1$ and $g(z) \neq 0$ for every $z$. Then there is a unique complex-valued function $f$ on $\mathbb{R}$ such that $f(0)=0$ and $\exp (f(z))=g(z)$. We write $f(z)=\log (g(z))$ and call $f$ the distinguished logarithm of $g$. In the case that $g$ is a characteristic function, then $f$ is called the cumulant function. 
Throughout the paper, we will work with homogeneous Lévy bases, which have a cumulant function of the form

$$
\begin{aligned}
\mathrm{C}(\zeta, L(A)) & =\log (\mathbb{E}[\exp (\mathrm{i} \zeta L(A))]) \\
& =\left[\mathrm{i} \zeta a-\frac{1}{2} \zeta^{2} b+\int_{\mathbb{R}}\left(\mathrm{e}^{\mathrm{i} \zeta z}-1-\mathrm{i} \zeta z \mathbf{1}_{[-1,1]}(z)\right) \nu(\mathrm{d} z)\right] \operatorname{leb}(A),
\end{aligned}
$$

where leb( $\cdot)$ denotes the Lebesgue measure, $a \in \mathbb{R}, b \geq 0$, and $v$ is a Lévy measure on $\mathbb{R}$. Hence, we can associate an infinitely divisible random variable $L^{\prime}$, called the Lévy seed, with every homogeneous Lévy basis $L$ such that, for $A \in \mathcal{B}_{d}\left(\mathbb{R}^{d}\right)$,

$$
\mathrm{C}(\zeta, L(A))=\operatorname{leb}(A) \mathrm{C}\left(\zeta, L^{\prime}\right)
$$

where

$$
C\left(\zeta, L^{\prime}\right)=\log \left(\mathbb{E}\left[\exp \left(\mathrm{i} \zeta L^{\prime}\right)\right]\right)=\mathrm{i} \zeta a-\frac{1}{2} \zeta^{2} b+\int_{\mathbb{R}}\left(\mathrm{e}^{\mathrm{i} \zeta z}-1-\mathrm{i} \zeta z \mathbf{1}_{[-1,1]}(z)\right) v(\mathrm{~d} z)
$$

We call $(a, b, v)$ the characteristic triplet associated with $L^{\prime}$ and $(a, b, v$, leb) the characteristic quadruplet associated with the homogeneous Lévy basis $L$.

\subsection{The model}

Let us now introduce our new modelling framework for the electricity futures curve dynamics. Note that throughout the paper we do not distinguish between forward and futures prices, but present a general framework which is applicable for modelling both forward and futures prices. In the following, we consider a market with finite time horizon $\left[0, T^{*}\right]$ for some $T^{*} \in(0, \infty)$.

Note that in energy markets, futures specify a delivery period rather than a time of delivery. Hence, we are interested in modelling the futures price at time $t \geq 0$ with delivery period $\left[T_{1}, T_{2}\right]$ for $t \leq T_{1} \leq T_{2} \leq T^{*}$ say. In order to account for the delivery period, we use the standard method, as, e.g. described in [17] and [22, Chapter 6], and model the (instantaneous) futures price $F(t, T)$, where $T \in\left[T_{1}, T_{2}\right]$ is interpreted as the (instantaneous) time of delivery. The corresponding model for the futures price with delivery period $\left[T_{1}, T_{2}\right]$ is then found to be

$$
F_{t}\left(T_{1}, T_{2}\right)=\frac{1}{T_{2}-T_{1}} \int_{T_{1}}^{T_{2}} F(t, T) \mathrm{d} T .
$$

That is, we simply average the model for the instantaneous futures price over the delivery period in order to have the futures price for a contract with delivery period.

In the following, we will focus on modelling the (instantaneous) futures price $F(t, T)$ and (3.3) can then be used to derive the futures price for a given delivery period.

\subsection{Modelling the instantaneous futures price}

Let $t \geq 0$ denote the current time, let $T \in\left[t, T^{*}\right]$ be the time of delivery, and let $x=T-t$ be the time to delivery of the (instantaneous) futures contract. Let us introduce our modelling assumptions next.

(A1) $L$ is a homogeneous, square-integrable Lévy basis on $\mathbb{R}^{2}$, which has zero mean; its characteristic quadruplet is denoted by $(a, b, v$, leb). Let $\mathbb{Q}$ denote the covariance measure associated with $L$ satisfying $\mathbb{Q}(\mathrm{d} \xi, \mathrm{d} s)=\left(b+\int_{\mathbb{R}} z^{2} v(\mathrm{~d} z)\right) \mathrm{d} \xi \mathrm{d} s$. To simplify the exposition, we assume throughout the paper that $\mathbb{Q}$ is standardised and given by $\mathbb{Q}(\mathrm{d} \xi, \mathrm{d} s)=\mathrm{d} \xi \mathrm{d} s$. 
(A2) Let $\left\{\mathcal{F}_{t}\right\}_{t \in\left[-T^{*}, T^{*}\right]}$ denote a filtration satisfying the usual conditions such that, for fixed $A \in \mathscr{B}_{b}\left(\left[0, T^{*}\right]\right)$, the process $(L(A, t))_{t \in\left[-T^{*}, T^{*}\right]}$ is a martingale with respect to that filtration.

(A3) The positive random field $\sigma=\sigma(\xi, s): \Omega \times \mathbb{R}^{2} \rightarrow(0, \infty)$ denotes the so-called stochastic volatility field and is assumed to be independent of the Lévy basis $L$.

(A4) The function $k:\left[0, T^{*}\right] \times\left[0, T^{*}\right] \times\left[-T^{*}, T^{*}\right] \rightarrow[0, \infty)$ denotes the so-called weight function.

(A5) For each $T \in\left[0, T^{*}\right]$, the random field $(\sigma(\xi, s))_{(\xi, s) \in\left[0, T^{*}\right] \times\left[-T^{*}, T^{*}\right]}$ is assumed to be predictable and the following integrability condition needs to hold:

$$
\mathbb{E}\left[\int_{\left[0, T^{*}\right] \times\left[-T^{*}, T^{*}\right]} k^{2}(T ; \xi, s) \sigma^{2}(\xi, s) \mathrm{d} \xi \mathrm{d} s\right]<\infty .
$$

(A6) We call the set

$$
A_{t}=\left[0, T^{*}\right] \times\left[-T^{*}, t\right]=\left\{(\xi, s): 0 \leq \xi \leq T^{*},-T^{*} \leq s \leq t\right\} \subseteq\left[0, T^{*}\right] \times\left[-T^{*}, T^{*}\right]
$$

the ambit set.

(A7) The deterministic integrable function $\Lambda:\left[0, T^{*}\right] \rightarrow(0, \infty)$ denotes a seasonality and trend function.

Definition 3.2. Let $0 \leq t \leq T \leq T^{*}$. Under assumptions (A1)-(A7), the futures price under the risk-neutral probability measure is defined as the ambit field given by

$$
F(t, T)=\Lambda(T)+\int_{A_{t}} k(T ; \xi, s) \sigma(\xi, s) L(\mathrm{~d} \xi, \mathrm{d} s) .
$$

Often we work with the Musiela parametrisation with $x=T-t$, and define $f_{t}(x)=F(t, x+t)$. Then

$$
f_{t}(x)=\Lambda(t+x)+\int_{A_{t}} k(x+t ; \xi, s) \sigma(\xi, s) L(\mathrm{~d} \xi, \mathrm{d} s) .
$$

Note that the stochastic integral with respect to the Lévy basis $L$ is well defined in the sense of Walsh [66], which extends classical Itô integration with respect to martingales to integration with respect to martingale measures. A detailed review of this integration concept in the context of ambit fields is given in [14] and [15].

Remark 3.3. Let us give a justification for the choice of the ambit set as $A_{t}=\left[0, T^{*}\right] \times$ $\left[-T^{*}, t\right]$, which is depicted in Figure 1. As already mentioned, we consider a market with finite time horizon $T^{*}$, i.e. all futures contracts expire before that time point. We are interested in modelling the futures market from today, i.e. $t=0$, up to $T^{*}$. We assume that today's observations are realisations of a random field which is obtained by integrating over the past, i.e.

$$
F(0, T)=\int_{A_{0}} k(T ; \xi, s) \sigma(\xi, s) L(\mathrm{~d} \xi, \mathrm{d} s) .
$$

Without loss of generality, we assume here that the boundary of the time horizon in the past is given by $-T^{*}$ (rather than by a different parameter, e.g. $-T^{* *}$ ), which simplifies the exposition. Since we follow a modelling approach based on a random field, our model permits consistency with the initial term structure $F(0, T)$ and does not need to be recalibrated multiple times; see, e.g. [43] for a discussion of this aspect. 


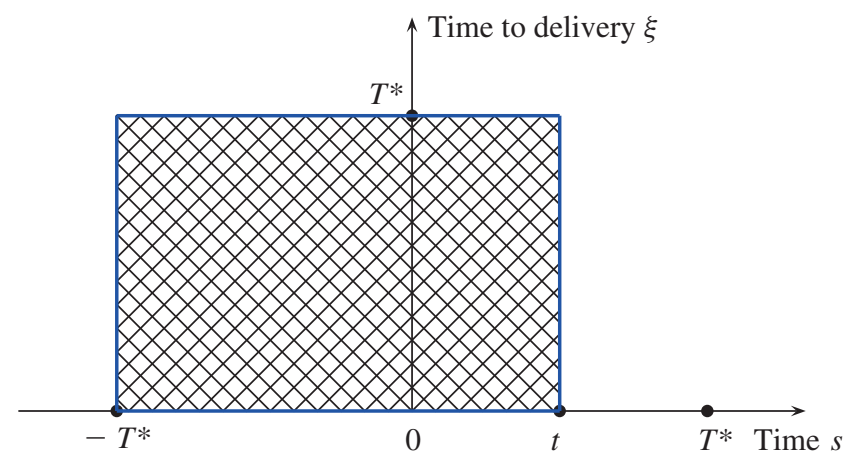

Figure 1: The ambit set $A_{t}=\left[0, T^{*}\right] \times\left[-T^{*}, t\right]$.

Remark 3.4. Note that the term ambit originates from the Latin words ambire and ambitus where the latter means border, boundary, or sphere of influence. Hence, ambit sets are the regions of influence in the past. We use the term ambit field to highlight that such random fields are constructed by integrating over ambit sets, which can be considered as describing the temporal past and the spatial neighbouring regions which are relevant for the object under investigation.

Our first result is that futures prices as defined in Definition 3.2 are martingales under the risk-neutral probability measure $\mathbb{Q}$.

Proposition 3.1. For $T \in\left[0, T^{*}\right]$, the stochastic process $(F(t, T))_{0 \leq t \leq T}$ is a martingale with respect to the filtration $\left\{\mathcal{F}_{t}\right\}_{t \in[0, T]}$.

Proof. The measurability and integrability are straightforward to show. Furthermore, for $0 \leq \tilde{t} \leq t$, we have $A_{\tilde{t}} \subseteq A_{t}$. Using the independence property of $\sigma$ and $L$ and the fact that $L$ is a zero-mean process, we find that

$$
\begin{aligned}
\mathbb{E}\left[F(t, T) \mid \mathcal{F}_{\tilde{t}}\right] & \\
& =\Lambda(T)+\mathbb{E}\left[\int_{A_{\tilde{t}}} k(T ; \xi, s) \sigma(\xi, s) L(\mathrm{~d} \xi, \mathrm{d} s)+\int_{A_{t} \backslash A_{\tilde{t}}} k(T ; \xi, s) \sigma(\xi, s) L(\mathrm{~d} \xi, \mathrm{d} s) \mid \mathcal{F}_{\tilde{t}}\right] \\
& =\Lambda(T)+\int_{A_{\tilde{t}}} k(T ; s, \xi) \sigma(\xi, s) L(\mathrm{~d} \xi, \mathrm{d} s) \\
& =F(\tilde{t}, T) .
\end{aligned}
$$

Alternatively, we could have argued as follows. The integrand in $F(t, T)$ does not depend on $t$ and satisfies the [66]-integrability conditions. Hence, the integral is itself a martingale measure, and, in addition, $\Lambda(T)$ does not depend on $t$, which implies that $(F(t, T))_{0 \leq t \leq T}$ is a martingale.

Remark 3.5. Note that necessary and sufficient conditions for a more general ambit field to be a martingale are discussed in Appendix A.

The important implication of Proposition 3.1 is that our modelling framework is free of arbitrage in the sense that there is no free lunch with vanishing risk as defined in [34].

We have chosen to model the futures price in (3.4) as an arithmetic model, which is in line with many recent developments in the literature. For example, Bernhardt et al. [23] and García 
et al. [39] proposed and argued statistically for an arithmetic spot price model for Singapore electricity data. An arithmetic spot model will naturally lead to arithmetic dynamics for the futures price. Benth et al. [21] proposed an arithmetic model for spot electricity, and derived arithmetic futures price dynamics. In [20] arithmetic spot and futures price models are used to investigate the risk premium theoretically and empirically for the German EEX market. See also [64] and [65] for recent work on arithmetic models for electricity spot and futures.

In addition, we will give a description of the corresponding geometric framework based on ambit fields in Section 5.

\subsection{Important properties of the new modelling framework}

The ambit field specification is highly analytically tractable. In particular, we can easily derive the (conditional) cumulant function.

Proposition 3.2. Let $g_{t}=\sigma\left\{\sigma(\xi, s),(\xi, s) \in A_{t}\right\}$. The conditional cumulant function of the futures price-given the $\sigma$-algebra generated by the stochastic volatility field-is given by

$$
\begin{aligned}
\mathrm{C}^{\sigma}\left(\zeta, f_{t}(x)\right) & :=\log \left(\mathbb{E}\left[\exp \left(\mathrm{i} \zeta f_{t}(x)\right) \mid g_{t}\right]\right) \\
& =\mathrm{i} \zeta \Lambda(t+x)+\int_{A_{t}} \mathrm{C}\left(\zeta k(x+t ; \xi, s) \sigma(\xi, s), L^{\prime}\right) \mathrm{d} \xi \mathrm{d} s,
\end{aligned}
$$

where $L^{\prime}$ is the Lévy seed associated with $L$ with cumulant function given by (3.2).

The proof of the above result is straightforward and given in, e.g. [15].

Example 3.1. If $L$ is a homogeneous Gaussian Lévy basis then we have

$$
\mathrm{C}\left(\zeta k(x+t ; \xi, s) \sigma(\xi, s), L^{\prime}\right)=\mathrm{i} \zeta \Lambda(t+x)-\frac{1}{2} \zeta^{2} k^{2}(x+t ; \xi, s) \sigma^{2}(\xi, s) .
$$

While models based on random fields have been previously studied in the literature, see [1], [43], [50], [51], [53], and [59], we stress that our model has an important property which most models previously proposed in the literature lack: it allows for stochastic volatility in an analytically tractable form. Note that Barndorff-Nielsen et al. [16], Benth [18], and Hikspoors and Jaimungal [46] provided empirical evidence that spot and futures prices are influenced by a stochastic volatility field $\sigma$. Here we assume that $\sigma$ describes the volatility of the futures market as a whole. The stochastic volatility is incorporated as a factor in the stochastic integrand, i.e. as the random field $\sigma(\xi, s)$. We typically use the quadratic variation to measure the accumulated 'stochastic volatility' over a certain time period. In our modelling context, we can deduce from [66, Theorem 2.5] that the quadratic variation of the futures price $f_{t}(x)$ is given by

$$
\int_{A_{t}} k^{2}(x+t ; \xi, s) \sigma^{2}(\xi, s) \mathrm{d} \xi \mathrm{d} s .
$$

Moreover, it is important to note that our new model does not only model one particular futures contract, but it models the entire futures curve at once. Hence, it is interesting to study the correlation structure for various futures contracts implied by our new modelling framework. For the particular model defined in (3.4), for $0 \leq t \leq t+h \leq T^{*}$ and $0 \leq x, x^{\prime} \leq T^{*}$, we have $A_{t} \cap A_{t+h}=A_{t}$ and

$$
\begin{aligned}
& \operatorname{cor}\left(f_{t}(x), f_{t+h}\left(x^{\prime}\right)\right) \\
& =\frac{\int_{A_{t}} k(x+t, \xi, s) k\left(x^{\prime}+t+h, \xi, s\right) \mathbb{E}\left[\sigma^{2}(\xi, s)\right] \mathrm{d} \xi \mathrm{d} s}{\sqrt{\int_{A_{t}} k^{2}(x+t, \xi, s) \mathbb{E}\left[\sigma^{2}(\xi, s)\right] \mathrm{d} \xi \mathrm{d} s \int_{A_{t+h}} k^{2}\left(x^{\prime}+t+h, \xi, s\right) \mathbb{E}\left[\sigma^{2}(\xi, s)\right] \mathrm{d} \xi \mathrm{d} s}} .
\end{aligned}
$$


Hence, we see that the correlation structure is determined by three factors: the intersection of the corresponding ambit sets (here we have $A_{t} \cap A_{t+h}=A_{t}$ ), the weight function, and the second moment of the stochastic volatility field. Furthermore, one could think of modelling various commodity forward or futures contracts, such as electricity and natural gas futures, simultaneously. In such a situation it becomes even more clear how flexible the ambit setup is. We can specify different ambit sets, weight functions, stochastic volatility fields, and Lévy bases, and obtain a rather flexible correlation structure. The details of these multivariate extensions can be found in [15].

\subsection{Examples of model specifications}

In order to construct a fully parametric model for the futures price, we need to specify the weight function $k$, the stochastic volatility field $\sigma(\xi, s)$, and the Lévy basis $L$. The choice of the model components will be based on stylised features, market intuition, and considerations of mathematical/statistical tractability. Also, we will need to impose parameter restrictions, which will ensure that the specific model parameters are indeed identifiable.

Since a futures model based on an ambit field has a very general structure, we would like to point out some concrete model specifications which might be useful in practice and are motivated from empirical work in [3], [16], and [25]. Clearly, in any particular application, the concrete specification should be determined in a data-relevant fashion.

3.5.1. Specification of the Lévy basis. Recall that we have defined our model based on a Lévy basis, which is square integrable and has zero mean. Extensions to allow for a nonzero mean are straightforward and, hence, omitted. In principle, we can choose any infinitely divisible distribution satisfying these two assumptions. A very natural starting point would be the Gaussian Lévy basis. Alternative interesting choices in the context of modelling financial data include the normal inverse gaussian (NIG) Lévy basis and the tempered stable Lévy basis.

3.5.2. Specification of the weight function. Next we present relevant specifications of the weight function $k$. Note that the weight function $k$ plays a key role for two reasons. First, it determines the tempospatial autocorrelation structure of our ambit field. Second, it characterises the Samuelson effect as we will see in Proposition 4.3 below.

Let us now study some relevant examples which are motivated by the recent literature. Note that many traditional models for futures prices are based on stochastic processes rather than on random fields and, hence, the corresponding weight function does not allow for a spatial parameter. In order to incorporate such models in our modelling framework, we could think of working under the following factorisation assumption for the weight function.

Assumption F. It holds that

$$
k(x+t ; \xi, s)=\Phi(\xi) \Psi(x+t, s)
$$

for suitable functions $\Psi$ and $\Phi$.

In the case that $\Phi \equiv 1$ (and that there is no stochastic volatility component) we would almost be back in the classical framework, as we will see in the following example.

Example 3.2. The traditional way to model the futures dynamics, using the Musiela parametrisation with $x=T-t$ in the [45] models, is given by

$$
\mathrm{d} f_{t}(x)=\frac{\partial f_{t}}{\partial x}(x) \mathrm{d} t+h(x, t) \mathrm{d} W_{t}
$$


where, for simplicity, we disregard any spatial dependency in the Gaussian field $W$ so that it is indeed a Brownian motion. Under appropriate (weak) conditions, the mild solution of this stochastic partial differential equation (SPDE) is given by

$$
f_{t}(x)=S_{t} f_{0}(x)+\int_{0}^{t} S_{t-s} h(x, s) \mathrm{d} W_{s},
$$

where $S_{t}$ is the right-shift operator, $S_{t} g(x)=g(x+t)$; see [28] and [33] for more details. Hence,

$$
f_{t}(x)=f_{0}(x+t)+\int_{0}^{t} h(s,(t+x)-s) \mathrm{d} W_{s}=f_{t}(T-t)=f_{0}(T)+\int_{0}^{t} h(s, T-s) \mathrm{d} W_{s} .
$$

In our new modelling framework, however, we are interested in nontrivial choices of $\Phi$ and $\Psi$. When working with a factorisation as in Assumption F, we could base our choice of the temporal weight function $\Psi$ on weight functions which have proven to be suitable in the case of modelling futures prices as a times series. Let us give some important examples in that context.

Example 3.3. Motivated by standard Ornstein-Uhlenbeck (OU) models, we could choose

$$
\Psi(x+t, s)=\exp (-\alpha(x+t-s))
$$

for some $\alpha>0$. The choice of $\Psi$ can also be motivated from continuous-time ARMA (CARMA) processes; see [26] and [27]. Specifically, for $\alpha_{i}>0, i=1, \ldots, p, p \in \mathbb{N}$, introduce the matrix

$$
\boldsymbol{A}=\left[\begin{array}{cc}
0 & \boldsymbol{I}_{p-1} \\
-\alpha_{p} & -\alpha_{p-1}-\cdots-\alpha_{1}
\end{array}\right]
$$

where $\boldsymbol{I}_{n}$ denotes the $n \times n$ identity matrix. For $0<p<q$, define the $p$-dimensional vector $\boldsymbol{b}^{\top}=\left(b_{0}, b_{1}, \ldots, b_{p-1}\right)$, where $b_{q}=1$ and $b_{j}=0$ for $q<j<p$, and introduce

$$
\Psi(x+t-s)=\boldsymbol{b}^{\top} \exp (\boldsymbol{A}(x+t-s)) \boldsymbol{e}_{p},
$$

with $\boldsymbol{e}_{k}$ being the $k$ th canonical unit vector in $\mathbb{R}^{p}$.

Another interesting example which does not belong to the class of linear models is given as follows.

Example 3.4. Motivated by a model introduced in [25], we could choose

$$
\Psi(x+t, s)=\frac{a}{x+t-s+b}
$$

for $a, b>0$.

We will come back to the latter example later on, when we focus in more detail on the Samuelson effect. Bjerksund et al. [25] suggested such a weight function since they found that it mimics the empirical Samuelson effect rather well.

Example 3.5. An important example is motivated by the model introduced in [3]. In addition to the choice $\Psi(x+t, s)=\exp (-\alpha(x+t-s))$ for $\alpha>0$, we could define $\Phi(\xi)=\exp (-\beta \xi)$ for $\beta>0$.

An extension of the above example would be to work with weights induced by linear CARMA models for the spatial component $\Phi$ also. 
Remark 3.6. It should be noted that our modelling framework allows for weight functions $k$ which are not separable in the $s$ and $\xi$ components, i.e. when Assumption F is not satisfied. This feature makes it possible to allow for much more sophisticated correlation structures than those used in classical models which are not based on random fields.

3.5.3. Specification of the stochastic volatility field. Let us briefly sketch possibilities of modelling the stochastic volatility field. First, there is the trivial choice of setting $\sigma \equiv 1$. However, given the empirical evidence of volatility clusters (at least in the time domain), it is unlikely that such a model will be suitable for an application to real data.

A natural starting point to construct a nontrivial stochastic volatility field would be to work with traditional stochastic volatility models which do not exhibit a spatial component. There are at least two reasons which would justify such a choice. First, from a practical point of view, we typically have very frequent data in the temporal dimension, but rather sparse data in the spatial dimension. This would suggest that, from a modelling point of view, we might want to focus on models which are not too complex in the spatial component. For example, it has been pointed out in the literature, see, e.g. [59], that we are often interested in very smooth models in the spatial component.

In Section 7 we will study a concrete example of a truly tempospatial volatility field. From a theoretical point of view, there are various ways to construct tempospatial stochastic volatility fields and we refer the reader to [15] for more results on this topic.

\subsection{Remark on how to include length of delivery in the model}

As mentioned before, the futures price corresponding to a delivery period $\left[T_{1}, T_{2}\right]$ is modelled by $F_{t}\left(T_{1}, T_{2}\right)=\left(T_{2}-T_{1}\right)^{-1} \int_{T_{1}}^{T_{2}} f_{t}(u-t) \mathrm{d} u$. Hence, given an ambit model of $f_{t}(x)$, we simply average it over the delivery period in order to have the futures price for a contract with delivery period.

Alternatively, we could think of modelling $F_{t}\left(T_{1}, T_{2}\right)$ directly-by an ambit field. The main idea here is to include the length of the delivery period $\tau:=T_{2}-T_{1}$ as an additional spatial component. For example, we could think of using

$$
\int_{A_{t}^{*}} k(x+t, \tau ; \xi, \chi, s) \sigma_{s}(\xi, \chi) L(\mathrm{~d} \xi, \mathrm{d} \chi, \mathrm{d} s),
$$

as a building block for $F_{t}\left(T_{1}, T_{2}\right)$, where $A_{t}^{*}$ is a suitably extended ambit set and where we assume that the corresponding integrability conditions hold, which ensure that the ambit field is well defined. The main obstacle in building such models is the noarbitrage condition between contracts with overlapping delivery periods. In fact, any model for $F_{t}\left(T_{1}, T_{2}\right)$ must satisfy (see [22])

$$
F_{t}\left(T_{1}, T_{2}\right)=\frac{1}{T_{2}-T_{1}} \int_{T_{1}}^{T_{2}} F_{t}(\tau, \tau) \mathrm{d} \tau,
$$

which puts serious restrictions on the degrees of freedom in modelling. It will be interesting to study the analytical properties of such models in more detail in future research.

\section{Constructing the spot model from the futures price}

After having studied the new model for the futures price, we investigate in detail the nature of the spot price model implied by our new modelling framework for the futures price. Recall that we are modelling under the risk-neutral measure. 


\subsection{The implied spot price}

By the noarbitrage assumption, the futures price for a contract which matures in zero time, $x=0$, has to be equal to the spot price, that is, $f_{t}(0)=S_{t}$. Thus,

$$
S_{t}=\Lambda(t)+\int_{A_{t}} k(t ; \xi, s) \sigma(\xi, s) L(\mathrm{~d} \xi, \mathrm{d} s)
$$

More precisely, we have the following result.

Proposition 4.1. Suppose that $f_{t}(0)=S_{t}$ and that

$$
\lim _{x \downarrow 0} \int_{A_{t}}(k(x+t ; \xi, s)-k(t ; \xi, s))^{2} \mathbb{E}\left[\sigma^{2}(\xi, s)\right] \mathrm{d} \xi \mathrm{d} s=0 .
$$

Then $f_{t}(x) \rightarrow S_{t}$ in $L^{2}(\mathbb{Q})$ as the time to maturity $x$ tends to 0 .

Proof. This follows readily by appealing to the Itô-type isometry for Walsh integrals.

The proposition shows us that the futures price will tend continuously in variance to the spot price as the time to maturity decreases to 0 .

We observe that the spot price process implied by our ambit field based futures price model is driven by a (tempospatial) Lévy basis and not just by a Brownian motion or a Lévy process. Barndorff-Nielsen et al. [16] proposed modelling energy spot prices by volatility modulated Volterra processes, and we observe that our implied spot price $S_{t}$ is in fact a superposition of such spot models, in the same sense as one has superpositions of OU processes. That is, we do not just integrate out the time parameter $s$, but we have an additional parameter $\xi$, which appears both in the weight function and in the stochastic volatility component and can be viewed as being randomised through the Lévy basis $L$.

Similarly to the result for the futures price, see Proposition 3.2, we can derive the conditional cumulant function for the implied spot price.

Proposition 4.2. Let $L$ be a homogeneous Lévy basis. Then, for $S_{t}$ as defined by (4.1), the conditional cumulant function is given by

$$
\mathrm{C}^{\sigma}\left(\zeta, S_{t}\right)=\log \left(\mathbb{E}\left[\exp \left(\mathrm{i} \zeta S_{t}\right) \mid g_{t}\right]\right)=\mathrm{i} \zeta \Lambda(t)+\int_{A_{t}} \mathrm{C}\left(\zeta k(t ; \xi, s) \sigma(\xi, s), L^{\prime}\right) \mathrm{d} \xi \mathrm{d} s,
$$

where $L^{\prime}$ is the Lévy seed associated with $L$.

A case of some special interest is the situation where the driving Lévy basis of the ambit field is a homogeneous Gaussian Lévy basis. Then we obtain the following result.

Corollary 4.1. In the Gaussian case, where $C\left(\zeta, L^{\prime}\right)=-\frac{1}{2} \zeta^{2}$,

$$
\mathrm{C}^{\sigma}\left(\zeta, S_{t}\right)=\mathrm{i} \zeta \Lambda(t)-\frac{1}{2} \zeta^{2} \int_{A_{t}} k^{2}(t ; s, \xi) \sigma_{s}^{2}(\xi) \mathrm{d} \xi \mathrm{d} s
$$

If $k$ factorises as in Assumption $\mathrm{F}$ then

$$
\mathrm{C}^{\sigma}\left(\zeta, S_{t}\right)=\mathrm{i} \zeta \Lambda(t)-\frac{1}{2} \zeta^{2} \int_{-T^{*}}^{t} \Psi^{2}(t ; s) \omega_{s}^{2} \mathrm{~d} s, \quad \text { where } \omega_{s}^{2}=\int_{0}^{T^{*}} \Phi^{2}(\xi) \sigma_{s}^{2}(\xi) \mathrm{d} \xi
$$


This implies that

$$
S_{t} \stackrel{\text { law }}{=} \int_{-T^{*}}^{t} \Psi(t ; s) \omega_{s} \mathrm{~d} W_{s},
$$

where $W$ is a Brownian motion. The latter is indeed a volatility modulated Volterra process which is driven by a Brownian motion. Such processes have been used as a model for energy spot prices in [16].

Remark 4.1. So far, we have assumed that $T^{*}<\infty$. Suppose for now that $\Psi(t, s)=\Psi(t-s)$, and consider the case in which $T^{*} \rightarrow \infty$. Then, provided the limit exists, the spot price is given by a Lévy semistationary process. This is indeed an interesting case since it includes many of the traditional models for electricity spot prices.

Note that it is well known that there is no convergence of electricity futures prices to the spot as time to start of delivery approaches. That is, if the delivery period is $\left[T_{1}, T_{2}\right], T_{1}<T_{2}$, then the futures price $F_{t}\left(T_{1}, T_{2}\right)$ at time $t$ does not converge to the spot price as $t \rightarrow T_{1}$. We say that we observe a risk premium; see, e.g. [64] for some recent work on this aspect. One could mimic such a behaviour with the model class we study here, by choosing the 'delivery time' $T$ as the midpoint, say, in the delivery interval $\left[T_{1}, T_{2}\right], T=\left(T_{1}+T_{2}\right) / 2$. Then we can still associate a spot price to the futures dynamics $f_{t}(x)$, but we will never actually observe the convergence in the market since at the start of delivery we have $x=\left(T_{2}-T_{1}\right) / 2$. On the other hand, we will obtain a model where there is an explicit connection between the futures at 'maturity' $t=T_{1}$ and the spot $S_{T_{1}}$. This allows for modelling spot and futures jointly, taking into account their dependency structure.

\subsection{Relation to the Samuelson effect}

Recall that the Samuelson effect describes the empirical fact that the volatility of the futures price increases when the time to delivery approaches 0 . In that case, from noarbitrage arguments, we also expect that (at least theoretically) the volatility of the futures converges to the volatility of the spot price. This finding is in fact naturally included in our modelling framework, which we will show in the following.

Recall that the weight function $k$ plays the role of a damping function and is therefore nonincreasing in the first variable. In particular, this means that the function $x \mapsto k(x+t ; \xi, s)$ is monotonically nonincreasing in $x \geq 0$ for every $(\xi, s) \in\left[0, T^{*}\right] \times\left[-T^{*}, T^{*}\right]$. Equivalently, we could say that $x \mapsto k(x+t ; \xi, s)$ is monotonically nondecreasing as $x \downarrow 0$.

Proposition 4.3. Assume that the function $x \mapsto k(x+t ; \xi, s)$ is monotonically nonincreasing in $0 \leq x$ for every $(\xi, s) \in\left[0, T^{*}\right] \times\left[-T^{*}, T^{*}\right]$. Let $c=\operatorname{var}\left(L^{\prime}\right)$, and assume that

$$
\sup _{(\xi, s) \in\left[0, T^{*}\right] \times\left[-T^{*}, T^{*}\right]} \mathbb{E}\left[\sigma^{2}(\xi, s)\right] \leq K \text { for a constant } K>0 .
$$

Then the variance of the futures price $f_{t}(x)$, given by

$$
v_{t}(x):=\operatorname{var}\left(f_{t}(x)\right)=c \int_{A_{t}} k^{2}(x+t ; \xi, s) \mathbb{E}\left[\sigma^{2}(\xi, s)\right] \mathrm{d} \xi \mathrm{d} s,
$$

is monotonically nondecreasing as $x \downarrow$. Furthermore, under the conditions of Proposition 4.1, the variance of the futures converges to the variance of the implied spot price.

Proof. Let $0 \leq x \leq x^{\prime}$. Then

$$
k^{2}\left(x^{\prime}+t ; \xi, s\right)-k^{2}(x+t ; \xi, s) \leq 0 .
$$


Hence,

$$
v_{t}\left(x^{\prime}\right)-v_{t}(x)=\int_{A_{t}}\left(k^{2}\left(x^{\prime}+t ; \xi, s\right)-k^{2}(x+t ; \xi, s)\right) \mathbb{E}\left[\sigma^{2}(\xi, s)\right] \mathrm{d} \xi \mathrm{d} s \leq 0,
$$

due to the fact that $k(x+t ; \xi, s)$ is nonincreasing in $x$.

Note that the variance of the spot is given by $v_{t}(0)$, and it follows from Proposition 4.1 that $v_{t}(x) \leq v_{t}(0)$ for $x \geq 0$. As $x \downarrow 0, v_{t}(x)$ is a monotonically nondecreasing sequence which is furthermore bounded by $v_{t}(0)$. Thus, there exists a $\operatorname{limit}_{x \downarrow 0} v_{t}(x) \leq v_{t}(0)$. Under the condition of Proposition 4.1, this limit will be $v_{t}(0)$, the spot price variance. That is, we have a Samuelson effect.

Example 4.1. Let us study the Samuelson effect in more detail in the absence of stochastic volatility and under the assumption that the weight function factorises as in Assumption F (and each component satisfies suitable integrability conditions). Then the variance of the futures price is given by

$$
v_{t}(x)=c^{\prime} \int_{-T^{*}}^{t} \Psi^{2}(x+t, s) \mathrm{d} s, \quad \text { where } \quad c^{\prime}=c \int_{0}^{T^{*}} \Phi^{2}(\xi) \mathrm{d} \xi .
$$

This implies that, in the context of Example 3.3 where we worked with an exponential weight, we obtain

$$
v_{t}(x)=c^{\prime} \frac{1}{2 \alpha}\left(\mathrm{e}^{-2 \alpha x}-\mathrm{e}^{-2 \alpha\left(x+t+T^{*}\right)}\right),
$$

and, in the context of the [25] model, see Example 3.4, we have

$$
v_{t}(x)=c^{\prime} a^{2}\left(\frac{1}{x+b}-\frac{1}{x+t+T^{*}+b}\right) .
$$

While in the first case the convergence to the spot volatility is determined by the exponential function, the ratio function determines the convergence in the second case.

Example 4.2. We now consider the limiting case of the example above when $T^{*} \rightarrow \infty$ (assuming that the corresponding integrals exists). Let us focus on the case of the model motivated by Bjerksund et al. [25], where we have

$$
k(x+t ; \xi, s)=\Phi(\xi) \frac{a}{x+t-s+b}, \quad a, b>0 .
$$

Then the variance of the futures price satisfies $v_{t}(x)=c^{\prime} a^{2} /(x+b)$, where we take $T^{*} \rightarrow \infty$ in the definition of $c^{\prime}$. Also, for the correlation between futures with different times to maturity, we obtain, for $h>0$,

$$
\operatorname{cor}\left(f_{t}(x), f_{t}(x+h)\right)=\log (1+r) \frac{\sqrt{1+r}}{r} \text { for } r=\frac{h}{x+b} .
$$

We observe that the correlation tends to 1 as $r \downarrow 0$, which corresponds to either $h \downarrow 0$, i.e. when we consider futures with the same time to maturity, or $x \rightarrow \infty$, i.e. futures in the long end are perfectly correlated. Also, the correlation tends to 0 as $r \rightarrow \infty$, corresponding to $h \rightarrow \infty$, meaning futures far apart from each other in terms of time of maturity are uncorrelated. Finally, we remark that the correlation is monotonically decreasing with $r$, or, equivalently, the correlation is increasing with time to maturity. This means that we have more idiosyncratic risk in the short end than in the long end of the market. 


\section{A geometric modelling framework}

So far, we have worked with an arithmetic model for the futures price since this is a very natural model choice and is in line with the traditional random field based models where the futures are directly modelled by, e.g. a Gaussian random field.

An alternative approach, which ensures that futures prices are always positive, would be to work with geometric models, i.e. we model the futures price as the exponential of an ambit field. Most of the results we derived before can be directly carried over to the geometric setup. For example, when we study the link between the futures price and the spot price, this has to be interpreted as the link between the logarithmic futures price and the logarithmic spot price. Likewise, when looking at probabilistic properties such as the moments and cumulants of the processes, they can be regarded as the moments/cumulants of the logarithmic futures price.

The only result, which indeed needs some adjustment, is in fact the martingale property. There will be an additional drift condition compared to the arithmetic case.

Before we formulate the martingale property, we specify an additional integrability assumption.

Assumption I. Suppose that the assumptions (A1)-(A7) are satisfied. In addition, we assume that

$$
\mathbb{E}\left[\exp \left(\int_{A_{t}} C\left(-\mathrm{i} k(T ; \xi, s) \sigma(\xi, s), L^{\prime}\right) \mathrm{d} \xi \mathrm{d} s\right)\right]<\infty \text { for all } t \in\left[0, T^{*}\right] .
$$

Now we show that a suitable specification of a geometric model based on ambit fields is a martingale.

Proposition 5.1. We assume that Assumption I is satisfied. Then the futures price at time $t \geq 0$ with delivery at time $T \in\left[t, T^{*}\right]$, denoted by $(G(t, T))_{t \in[0, T]}$ with

$$
\begin{aligned}
& G(t, T) \\
& \quad=\exp \left(\Lambda(T)+\int_{A_{t}} k(T ; \xi, s) \sigma(\xi, s) L(\mathrm{~d} \xi, \mathrm{d} s)-\int_{A_{t}} C\left(-\mathrm{i} k(T ; \xi, s) \sigma(\xi, s), L^{\prime}\right) \mathrm{d} \xi \mathrm{d} s\right),
\end{aligned}
$$

is a martingale with respect to $\left\{\mathcal{F}_{t}\right\}_{t \in[0, T]}$.

Proof. This results follows immediately from the results corresponding to the Esscher transform for semimartingales, see [49], and from our integrability condition, Assumption I.

Example 5.1. In the special case where $L=W$ is a standardised, homogeneous Gaussian Lévy basis and Assumption I is satisfied, $(G(t, T))_{t \in[0, T]}$ with

$$
G(t, T)=\exp \left(\Lambda(T)+\int_{A_{t}} k(T ; \xi, s) \sigma(\xi, s) W(\mathrm{~d} \xi, \mathrm{d} s)-\frac{1}{2} \int_{A_{t}} k^{2}(T ; \xi, s) \sigma^{2}(\xi, s) \mathrm{d} \xi \mathrm{d} s\right)
$$

is a martingale with respect to $\left\{\widetilde{F}_{t}\right\}_{t \in[0, T]}$.

\section{Option pricing}

Next we describe how to price options based on futures contracts with price dynamics given by an ambit field. Recall that the futures price at time $t \geq 0$ of a contract maturing at time $T \in\left[t, T^{*}\right]$ is —in the arithmetic model—given by

$$
f_{t}(T-t)=\Lambda(T)+\int_{A_{t}} k(T ; \xi, s) \sigma(\xi, s) L(\mathrm{~d} \xi, \mathrm{d} s)
$$


which is a martingale in $t$. Given a measurable function $g: \mathbb{R} \mapsto \mathbb{R}$, consider the problem of pricing a European option which pays $g\left(f_{\tau}(T-\tau)\right)$ at exercise time $\tau \leq T$. From the arbitrage theory, we find that the price of this option at time $t \leq \tau$ is

$$
C(t)=\mathrm{e}^{-r(\tau-t)} \mathbb{E}\left[g\left(f_{\tau}(T-\tau)\right) \mid \mathcal{F}_{t}\right] .
$$

Here the constant $r>0$ is the risk-free interest rate. For $C$ to be well defined, we must suppose that $g\left(f_{\tau}(T-\tau)\right)$ is integrable.

Since the cumulant function of the ambit field is available (see (3.5)), the Fourier-based pricing method is an attractive approach (see [29]). If $g, \hat{g} \in L^{1}(\mathbb{R})$, with $\hat{g}$ being the Fourier transform of $g$, we can express the price of the option as

$$
C(t)=\frac{1}{2 \pi} \int_{\mathbb{R}} \hat{g}(z) \mathbb{E}\left[\mathrm{e}^{\mathrm{i} z f_{\tau}(T-\tau)} \mid \mathcal{F}_{t}\right] \mathrm{d} z .
$$

Here we make use of the integral representation of the inverse Fourier transform; see [37]. Thus, to find $C(t)$, we must compute the conditional cumulant function of $Y$. First, we split the ambit field to obtain

$$
f_{\tau}(T-\tau)=\Lambda(T)+\int_{A_{t}} k(T ; s, \xi) \sigma_{s}(\xi) L(\mathrm{~d} \xi, \mathrm{d} s)+\int_{A_{\tau} \backslash A_{t}} k(T ; s, \xi) \sigma_{s}(\xi) L(\mathrm{~d} \xi, \mathrm{d} s) .
$$

The first integral on the right-hand side is $\mathcal{F}_{t}$-measurable. Hence,

$$
\begin{aligned}
\mathbb{E}\left[\mathrm{e}^{\mathrm{i} z f_{\tau}(T-\tau)} \mid \mathcal{F}_{t}\right]= & \exp \left(\mathrm{i} z \Lambda(T)+\mathrm{i} z \int_{A_{t}} k(T ; s, \xi) \sigma_{s}(\xi) L(\mathrm{~d} \xi, \mathrm{d} s)\right) \\
& \times \mathbb{E}\left[\exp \left(\mathrm{i} z \int_{A_{\tau} \backslash A_{t}} k(T ; s, \xi) \sigma_{s}(\xi) L(\mathrm{~d} \xi, \mathrm{d} s)\right) \mid \mathcal{F}_{t}\right] .
\end{aligned}
$$

The conditional expectation can be expressed analogously as in (3.5). Note that the option price will not depend explicitly on $f_{t}(T-t)$.

Many relevant payoff functions $g$ will not be in $L^{1}(\mathbb{R})$. For example, the payoff of a call option $g(x)=\max (x-K, 0)$ will fail to satisfy this condition. In such circumstances, one can dampen the payoff function by some exponential, and use the same procedure as above (see [29] for more details, including examples). In the case of geometric futures price models, we apply the machinery above to the payoff function $h(x)=g(\exp (x))$.

\section{Simulation study}

In this section we will describe how to simulate an ambit field of the form

$$
Y_{t}(x):=\int_{\left[0, T^{*}\right] \times\left[-T^{*}, t\right]} k(x+t ; \xi, s) \sigma(\xi, s) L(\mathrm{~d} \xi, \mathrm{d} s),
$$

where we will assume that the volatility field is given by

$$
\sigma^{2}(\xi, s)=V\left(\int_{\left[0, T^{*}\right] \times\left[-T^{*}, s\right]} j(\xi, s ; v, u) L^{\sigma}(\mathrm{d} v, \mathrm{~d} u)\right),
$$

where $V$ is a positive function, $L^{\sigma}$ is a Lévy basis which is independent of $L$, and the positive weight function $j$ is such that the integral in (7.1) is well defined.

In order to obtain our model for the futures price, we will later add a seasonality component. 


\subsection{Simulation algorithm}

We begin by constructing a grid for the interval $\left[0, T^{*}\right] \times\left[-T^{*}, T^{*}\right]$ by dividing the temporal dimension $\left[-T^{*}, T^{*}\right]$ into $n-1$ equidistant intervals of length $\Delta_{t}=2 T^{*} / n$, where we write $-T^{*}=t_{1}<t_{2}<\cdots<t_{n}=T^{*}$, and by dividing the spatial dimension $\left[0, T^{*}\right]$ into $m-1$ equidistant intervals of length $\Delta_{x}=T^{*} / m$, where we write $0=x_{1}<x_{2}<\cdots<x_{m}=T^{*}$ for $n, m \in \mathbb{N}$.

1. Simulate the stochastic volatility field on the grid points $\left(x_{j}, t_{i}\right)$ for $i=1, \ldots, n$ and $j=1, \ldots, m$. We obtain the values $\sigma\left(x_{j}, t_{i}\right)$. In the absence of stochastic volatility, we set $\sigma\left(x_{j}, t_{i}\right)=1$ for all $i, j$.

2. Set $\Delta:=\Delta_{x} \Delta_{t}$ and simulate $n(m-1)$ independent random variables $Z_{1, J} \stackrel{\mathrm{D}}{=} L\left(\Delta_{x}\right)$ and $Z_{I, J} \stackrel{\mathrm{D}}{=} L(\Delta)$ for $I=2, \ldots, n$ and $J=1, \ldots, m-1$.

3. For $j=1, \ldots, m$, we set

$$
\widehat{Y}_{t_{1}}\left(x_{j}\right)=\sum_{J=1}^{m-1} k\left(x_{j}+t_{1} ; x_{J}, t_{1}\right) \sigma\left(x_{J}, t_{1}\right) Z_{1, J} .
$$

4. For $i=2, \ldots, n$ and $j=1, \ldots, m$, we set

$$
\widehat{Y}_{t_{i}}\left(x_{j}\right):=\sum_{I=1}^{i-1} \sum_{J=1}^{m-1} k\left(x_{j}+t_{i} ; x_{J}, t_{I},\right) \sigma\left(x_{J}, t_{I}\right) Z_{I, J} .
$$

Steps 3 and 4 of the simulation algorithm make use of the definition of the stochastic integral in the sense of [66] for simple processes. This allows us to represent the stochastic integral of $Y_{t}(x)$ as $\widehat{Y}_{t}(x)$ in a discretised form, ensuring convergence when $\Delta$ goes to 0 .

In the presence of stochastic volatility, we can use the same procedure as described above for simulating the stochastic volatility field first.

Remark 7.1. Note that $\Delta=\operatorname{leb}\left(\left[x_{j}, x_{j+1}\right] \times\left[t_{i}, t_{i+1}\right]\right)$. In the case that $L=W$ is a Gaussian homogeneous Lévy basis, with characteristic quadruplet $\left(\mu, c^{2}, 0\right.$, leb), we simulate $Z_{I, J} \sim$ independent and identically distributed $N\left(\mu \Delta, c^{2} \Delta\right)$.

\subsection{A recursive scheme for an exponential weight function}

With this general simulation algorithm at hand, let us consider a specific example, where we can derive a recursive scheme which allows us to simulate the ambit field much more quickly.

Let $A_{t}=\left[0, T^{*}\right] \times\left[-T^{*}, t\right]$, and suppose that the weight function $k$ is a weighted sum of two exponential functions, i.e.

$$
k(x+t ; \xi, s)=w \exp \left(-\lambda_{1}(x+t-s+\xi)\right)+(1-w) \exp \left(-\lambda_{2}(x+t-s+\xi)\right)
$$

for $w \in[0,1]$ and $\lambda_{1}, \lambda_{2}>0$. This choice of weight function is motivated by the empirical studies in [16], where such a specification was shown to fit spot price data collected from the German power exchange EEX. In addition, suppose that $\sigma(\xi, s) \equiv 1$ for all $(\xi, s) \in$ $\left[0, T^{*}\right] \times\left[-T^{*}, T^{*}\right]$. Owing to the specification of the weight function, we can split the ambit field into two parts, i.e.

$$
Y_{t}(x)=w Y_{t}^{(1)}(x)+(1-w) Y_{t}^{(2)}(x)
$$


where

$$
Y_{t}^{(i)}(x)=\int_{A_{t}} \mathrm{e}^{-\lambda_{i}(x+t-s+\xi)} L(\mathrm{~d} \xi, \mathrm{d} s)=\mathrm{e}^{-\lambda_{i}(t+x)} \int_{A_{t}} \mathrm{e}^{-\lambda_{i}(\xi-s)} L(\mathrm{~d} \xi, \mathrm{d} s)
$$

for $i=1,2$. We immediately see that, for $\Delta_{t}>0$,

$$
Y_{t+\Delta_{t}}^{(i)}(x)=\mathrm{e}^{-\lambda_{i} \Delta_{t}} Y_{t}^{(i)}(x)+\mathrm{e}^{-\lambda_{i} \Delta_{t}} \mathrm{e}^{-\lambda_{i}(t+x)} \int_{t}^{t+\Delta_{t}} \int_{0}^{T^{*}} \mathrm{e}^{-\lambda_{i}(\xi-s)} L(\mathrm{~d} \xi, \mathrm{d} s) .
$$

For small $\Delta_{t}$, the last integral can be approximated by

$$
\int_{t}^{t+\Delta_{t}} \int_{0}^{T^{*}} \mathrm{e}^{-\lambda_{i}(\xi-s)} L(\mathrm{~d} \xi, \mathrm{d} s) \approx \mathrm{e}^{\lambda_{i} t} \int_{0}^{T^{*}} \mathrm{e}^{-\lambda_{i} \xi} L\left(\mathrm{~d} \xi \times \Delta_{t}\right),
$$

to obtain the iterative Euler-like time-stepping scheme

$$
Y_{t+\Delta_{t}}^{(i)}(x) \approx \mathrm{e}^{-\lambda_{i} \Delta_{t}} Y_{t}^{(i)}(x)+\mathrm{e}^{-\lambda_{i}\left(x+\Delta_{t}\right)} \int_{0}^{T^{*}} \mathrm{e}^{-\lambda_{i} \xi} L\left(\mathrm{~d} \xi \times \Delta_{t}\right) .
$$

The integral over $\xi$ can be computed numerically using a Riemann-like approximation as in the general case above. We note that we can iterate numerically over space as well, since, for $\Delta_{x}$, we have the equality

$$
Y_{t}^{(i)}\left(x+\Delta_{x}\right)=\mathrm{e}^{-\lambda_{i} \Delta_{x}} Y_{t}^{(i)}(x) .
$$

We make use of (7.2) and (7.3) to implement efficient numerical schemes for the simulation of the whole field $Y_{t}(x)$.

\subsection{Examples}

First, we illustrate the effect of the choice of the weight function. We simulate a Gaussian ambit field and compare four choices of the kernel function.

1. An exponential weight function, i.e. $k(x+t ; \xi, s)=\exp \left(-\lambda_{1}(x+t-s+\xi)\right)$ with $\lambda_{1}=0.226$; see Figure 2(a).

2. A sum of two exponentials, i.e. $k(x+t ; \xi, s)=(1-w) \exp \left(-\lambda_{1}(x+t-s+\xi)\right)+$ $w \exp \left(-\lambda_{2}(x+t-s+\xi)\right)$ with $\lambda_{1}=0.226, \lambda_{2}=0.012$, and $w=0.07$; see Figure 2(b).

3. A Bjerksund et al. [25]-type weight function, i.e. $k(x+t ; \xi, s)=1 /(t+x-s+b+\xi)$ with $b=0.01$; see Figure 2(c).

4. A gamma-type weight function, i.e. $k(x+t ; \xi, s)=(x+t-s+b+\xi)^{v-1} \exp \left(-\frac{1}{2} \alpha(T-\right.$ $s+b+\xi))$ with $b=0.01, \alpha=0.055$, and $v=0.672$; see Figure 2(d).

In Figure 2 we present the corresponding ambit fields, which do not involve stochastic volatility and where the seasonality component is set to 0 for now. One can clearly see the differences between the four random fields. While Figure 2(a) decays rather quickly to 0 as $x \rightarrow T^{*}$, we see that this is not the case any more when we add a more slowly decaying second exponential weight function in Figure 2(b). The choice of the kernel function in Figure 2(c) is motivated by the work of Bjerksund et al. [25], who suggested that their weight function mimics the empirical Samuelson effect better than the exponential function. We observe that such a weight function can be considered as a special case of a gamma-type kernel, and show a 


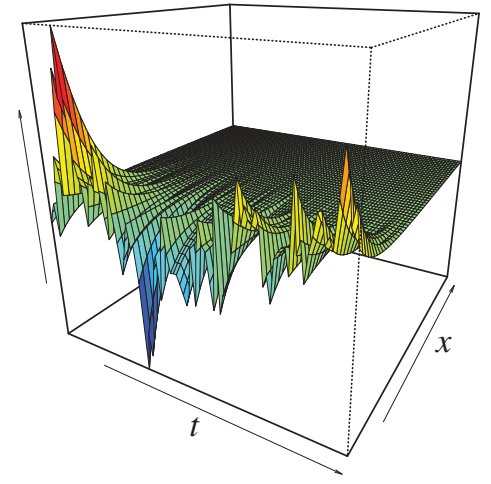

(a) Exponential weight function

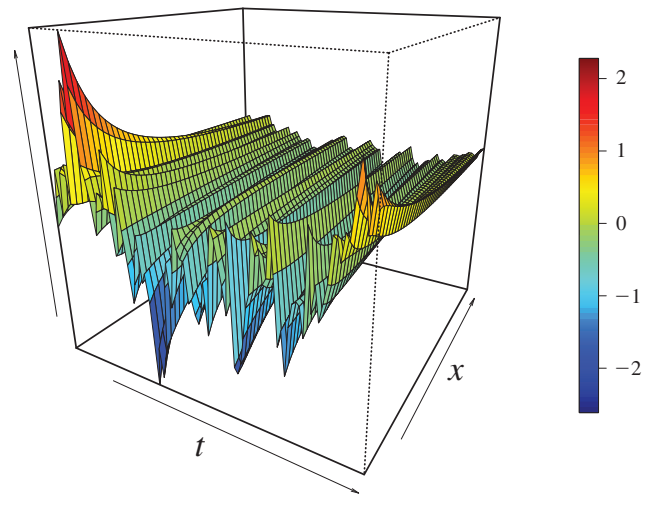

(c) Bjerksund et al.-type weight function

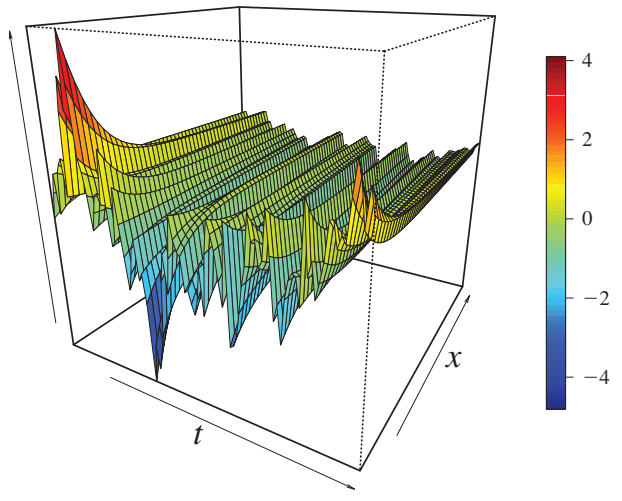

(b) Sum of two exponential weight functions

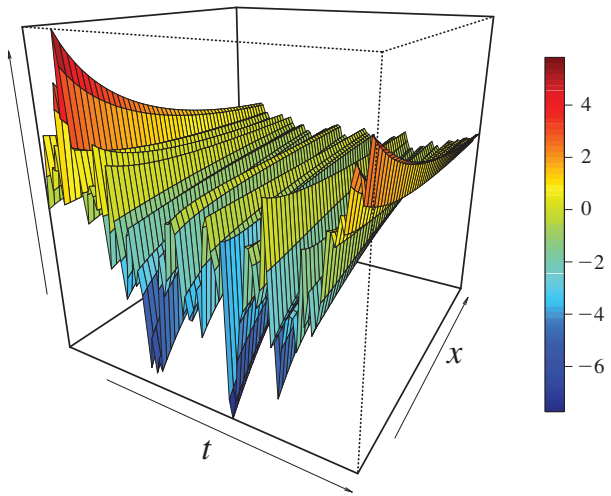

(d) Gamma-type weight function

FIGURE 2: Gaussian ambit field with four different choices for the kernel function.

realisation of such an ambit field in Figure 2(d). While the underlying Gaussian Lévy basis is the same in all four cases, we see very clearly that the choice of the weight function plays a key role in practice. In addition, we see that our model easily incorporates wide fluctuations in the short end and, in particular, in the implied spot price when $x=0$. At the same time, choosing a weight function which damps down the random noise as $x \rightarrow T^{*}$ leads to a rather smooth long end of the futures curve.

Let us now make our example more practically relevant by adding stochastic volatility as well as a seasonal component; see Figure 3. In the following, we choose only one weight function, namely the sum of two exponential functions with the same parameter choices as before. A realisation of such a field is depicted in Figure 3(a). Next we simulate a stochastic volatility field, where we suppose that $L^{\sigma}$ is an inverse Gaussian (IG) Lévy basis with mean $\varpi$ and variance $\varpi^{3} \varphi$, where we chose $\varpi=2$ and $\varphi=\varpi^{-3}$. Furthermore, $V$ being the identity function and $j$ being the exponential function, we have $j(s-u ; \xi, v)=\exp \left(-\lambda^{\sigma}(\xi+s-\right.$ $u+v)$ ) for $\lambda^{\sigma}>0$. Since the weight function $j$ is of exponential type, we can use the same considerations as above to obtain a time and space iterative scheme for simulation of $\sigma(x, t)$. A realisation of the volatility field is shown in Figure 3(b), where we have chosen the decay rate $\lambda^{\sigma}=0.5$ as an example. Note that this stochastic volatility process is a spatial generalisation 


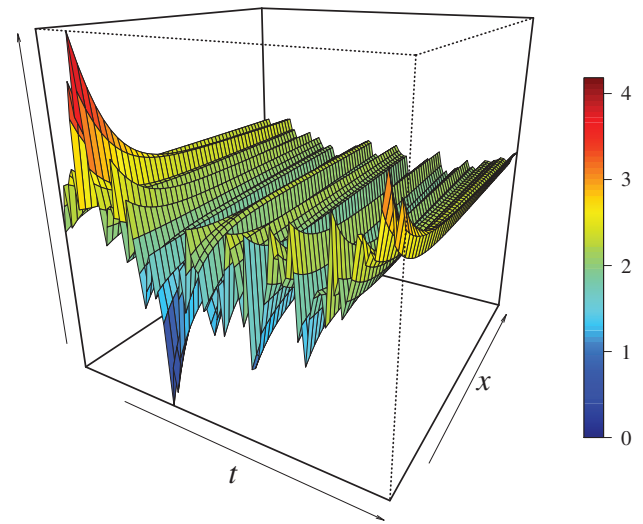

(a) Ambit field without stochastic volatility

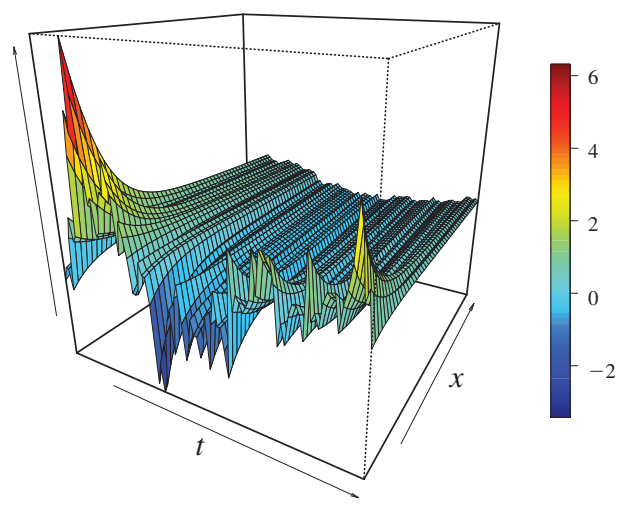

(c) Ambit field with stochastic volatility

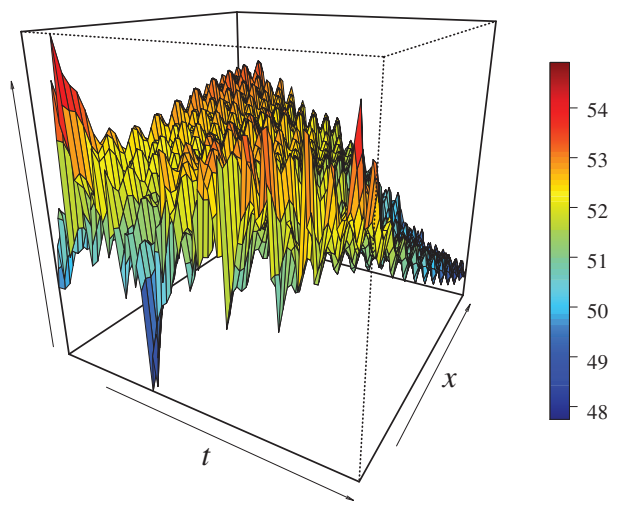

(e) Futures price without stochastic volatility

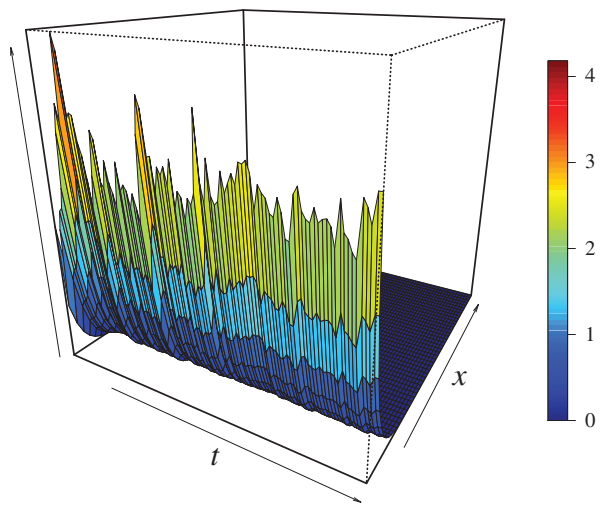

(b) Stochastic volatility field

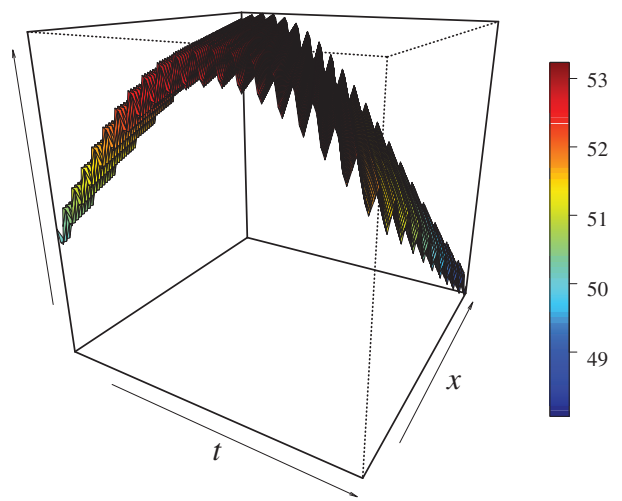

(d) Seasonality field

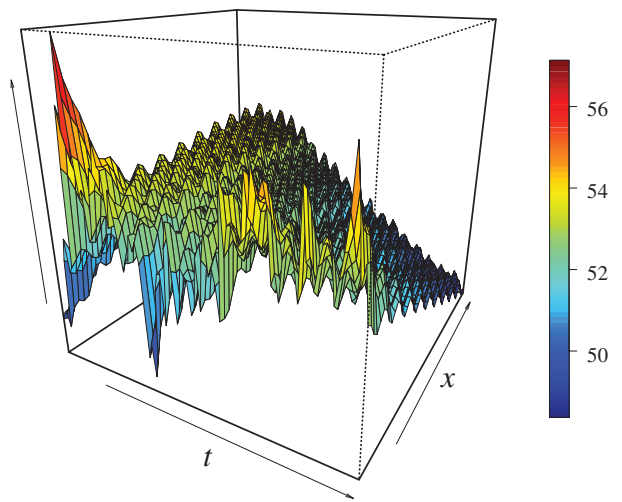

(f) Futures price with stochastic volatility

FIGURE 3: Building an arithmetic model for the futures prices from a Gaussian ambit field by accounting for seasonality and stochastic volatility. 
of a so-called Barndorff-Nielsen and Shephard (BNS) model, see [12], for the volatility process

$\sigma_{t}^{2}$, defined as the stationary solution of an IG Lévy process driven OU dynamics. The choice of speed of mean reversion equal to 0.5 will in that context yield a half-life of $\ln 2 / 0.5 \approx 1.4$, meaning that the volatility is fast mean reverting. This is in accordance with the empirical results found in [16]. The corresponding volatility modulated ambit field is then depicted in Figure 3(c). As we observe, the stochastic volatility case generates higher variation in the spot prices and the short end of the futures curve. This is due to the integration over the volatility in space that creates a higher variation than the Gaussian ambit field model. Also, since the stochastic volatility is chosen to be fast decaying to 0 as $x \rightarrow T^{*}$, in the long end the volatility smoothes the field compared to the ambit field without stochastic volatility in Figure 3(a). Clearly, different choices of the volatility field would be possible. Next, we would like to include seasonality in the futures curve, and add the seasonality field

$$
\Lambda(t+x)=50+3 \sin \left(\frac{2 \pi(t+x)}{250}\right)+0.25 \sin \left(\frac{2 \pi(t+x)}{5}\right),
$$

which accounts for yearly and weekly effects. Adding the seasonality field to the original ambit fields depicted in Figures 3(a) and 3(c) leads to our simulated futures curves depicted in Figures 3(e) and 3(f), where the former does not account for stochastic volatility whereas the latter does.

In our simulation study we have illustrated graphically that the class of ambit fields is very flexible and allows us to account for the specific stylised facts of electricity futures. We clearly see the influence of the seasonality function in the spot (i.e. for $x=0$ ), whereas in the long end it is essentially only the seasonality function that gives variations in the price. It should be noted that our simulation shows the instantaneous futures curve. Integrating over the corresponding delivery periods will smooth the field further in the $x$ direction and will then result in a curve reflecting the futures contracts with various delivery periods. This means that when considering delivery periods, the averaging will smoothen out much of the seasonality, at least for contracts with longer delivery periods. Typically, in the long end of the futures market we have yearly contracts, where the seasonality will be completely averaged out.

\section{Change of measure}

Since our futures price model is formulated under a risk-neutral pricing measure, it is of interest to understand how to get back to the physical measure in order to have a model which can be used for risk management purposes. We will introduce an Esscher transform to accommodate this.

Proposition 8.1. Define the process

$$
M_{t}^{\theta}=\exp \left(\int_{A_{t}} \theta(\xi, s) L(\mathrm{~d} \xi, \mathrm{d} s)-\int_{A_{t}} C\left(-\mathrm{i} \theta(\xi, s), L^{\prime}\right) \mathrm{d} \xi \mathrm{d} s\right) .
$$

The deterministic function $\theta:\left[0, T^{*}\right] \times\left[-T^{*}, T^{*}\right] \mapsto \mathbb{R}$ is supposed to be integrable with respect to the Lévy basis $L$ in the sense of [66]. Assume that

$$
\mathbb{E}\left[\exp \left(\int_{A_{t}} C\left(-\mathrm{i} \theta(s, \xi), L^{\prime}\right) \mathrm{d} \xi \mathrm{d} s\right)\right]<\infty \quad \text { for all } t \in \mathbb{R}_{T^{*}} .
$$

Then $M_{t}^{\theta}$ is a martingale with respect to $\mathcal{F}_{t}$ with $\mathbb{E}\left[M_{0}^{\theta}\right]=1$. 
The proof of the previous proposition is straightforward and, hence, omitted. We use that result now in order to define an equivalent probability $\mathbb{P}$ by

$$
\left.\frac{\mathrm{d} \mathbb{P}}{\mathrm{d} \mathbb{Q}}\right|_{\mathcal{F}_{t}}=M_{t}^{\theta}
$$

for $t \geq 0$. Hence, we have a change of measure from the risk-neutral probability $\mathbb{Q}$ under which the futures price is defined to a real-world probability $\mathbb{P}$. In effect, the function $\theta$ is an additional parameter to be modelled and estimated, and it will play the role as the market price of risk, as it models the difference between the risk-neutral and objective price dynamics.

We compute the characteristic exponent of an integral of $L$ under $\mathbb{P}$.

Proposition 8.2. For any $v \in \mathbb{R}$, and Walsh-integrable function $g$ with respect to $L$, it holds that

$$
\begin{aligned}
C_{P}\left(v, \int_{A_{t}} g(\xi, s) L(\mathrm{~d} \xi, \mathrm{d} s)\right) \\
\quad=\log \mathbb{E}_{\mathbb{P}}\left[\exp \left(\mathrm{i} v \int_{A_{t}} g(\xi, s) L(\mathrm{~d} \xi, \mathrm{d} s)\right)\right] \\
\quad=\log \mathbb{E}\left[\exp \left(\int_{A_{t}}(\mathrm{i} v g(\xi, s)+\theta(\xi, s)) L(\mathrm{~d} \xi, \mathrm{d} s)\right)\right] \exp \left(-\int_{A_{t}} C\left(-\mathrm{i} \theta(\xi, s), L^{\prime}\right) \mathrm{d} \xi \mathrm{d} s\right) \\
\quad=\int_{A_{t}}\left(C\left(v g(\xi, s)-i \theta(\xi, s), L^{\prime}\right)-C\left(-\mathrm{i} \theta(\xi, s), L^{\prime}\right)\right) \mathrm{d} \xi \mathrm{d} s .
\end{aligned}
$$

Note that the transform above is a simple generalisation of the Esscher transform of Lévy processes; see [13], [22], and [62] for more details on this aspect.

\section{Conclusion}

In this paper we presented a new modelling framework for electricity futures prices which followed the HJM methodology by modelling futures directly. We proposed using ambit fields, which are special types of random field, as the building block for the new modelling class. Ambit fields are constructed by stochastic integration with respect to Lévy bases using the integration concept of [66]. This ensured that the futures price was a martingale under the risk-neutral measure and, hence, we obtained a model free of arbitrage. We discussed relevant examples of model specifications within the new modelling framework and related them to the traditional modelling concepts. We showed that our new modelling framework accounts for the key stylised facts observed in electricity futures. Furthermore, we showed that futures and spot prices can be linked to each other within the ambit field framework. In addition, we discussed how a change of measure between the risk-neutral and physical probability measure can be carried out, so that our model can be used both for option pricing purposes as well as for applications in risk management under the physical probability measure.

A natural next step to take is to test our new model empirically and to study statistical aspects related to our ambit field models, such as model estimation and model specification tests.

\section{Appendix A. Martingale condition}

Recall that we have formulated the model for the futures price such that the price is a martingale. In the following, we formulate a martingale condition for more general ambit fields. 
Theorem A.1. Let $A_{t}=B \times\left[-T^{*}, t\right]$ for a bounded interval $B \subset \mathbb{R}$ and $T^{*}>0$, and define

$$
Y_{t}(T-t)=\int_{A_{t}} h(T-t, t ; \xi, s) \sigma(\xi, s) L(\mathrm{~d} \xi, \mathrm{d} s)
$$

for a deterministic weight function $h$, a nonnegative random field $\sigma$, and a homogeneous, square-integrable Lévy basis L with zero mean. Here we assume that the integrand satisfies the [66]-integrability conditions, and that $\sigma$ and $L$ are independent.

For every $T$, the necessary and sufficient condition for $\left(Y_{t}(T-t)\right)_{t \leq T}$ to be a continuous $L^{2}$-martingale with respect to the filtration defined in $(A 2)$ is that there exists a null set $N \in \mathbb{R}^{2}$ such that $t \mapsto h(T-t, t ; \xi, s)$ is a constant for all $(\xi, s) \notin N$.

Proof. The sufficiency of the condition is an immediate consequence of the definition of the [66]-integral. Hence, we focus on proving that the condition is also necessary. Suppose that $\left(Y_{t}(T-t)\right)_{t \leq T}$ is a continuous $L^{2}$-martingale. This implies in particular that

$$
\mathbb{E}\left[Y_{t}(T-t) \mid \mathcal{F}_{\tilde{t}}\right]=Y_{\tilde{t}}(T-\tilde{t}) \text { for all } \tilde{t} \leq t .
$$

Note that, for $\tilde{t} \leq t$, we have $A_{\tilde{t}} \subseteq A_{t}$. Using the independence property of $\sigma$ and $L$, and the fact that $L$ is a zero-mean process, we find that

$$
\begin{aligned}
\mathbb{E}\left[Y_{t}(T-t) \mid \mathcal{F}_{\tilde{t}}\right]=\mathbb{E}\left[\int_{A_{\tilde{t}}} h(T-t, t ; \xi, s) \sigma(\xi, s) L(\mathrm{~d} \xi, \mathrm{d} s)\right. \\
\left.\quad+\int_{A_{t} \backslash A_{\tilde{t}}} h(T-t, t ; \xi, s) \sigma(\xi, s) L(\mathrm{~d} \xi, \mathrm{d} s) \mid \mathcal{F}_{\tilde{t}}\right] \\
=\int_{A_{\tilde{t}}} h(T-t, t ; \xi, s) \sigma(\xi, s) L(\mathrm{~d} \xi, \mathrm{d} s) \\
=Y_{\tilde{t}}(T-\tilde{t})+I_{\tilde{t}}(T-\tilde{t}),
\end{aligned}
$$

where

$$
I_{\tilde{t}}(T-\tilde{t})=\int_{A_{\tilde{t}}}\{h(T-t, t ; \xi, s)-h(T-\tilde{t}, \tilde{t} ; \xi, s)\} \sigma(\xi, s) L(\mathrm{~d} \xi, \mathrm{d} s) .
$$

Without loss of generality, we assume that $\operatorname{var}\left(L^{\prime}\right)=1$. Since $L$ is a Lévy basis with zero mean, we know that $\mathbb{E}\left(I_{\tilde{t}}(T-\tilde{t})\right)=0$, and from the Itô isometry we therefore obtain

$$
\operatorname{var}\left(I_{\tilde{t}}(T-\tilde{t})\right)=\int_{A_{\tilde{t}}}\{h(T-t, t ; \xi, s)-h(T-\tilde{t}, \tilde{t} ; \xi, s)\}^{2} \mathbb{E}\left(\sigma^{2}(\xi, s)\right) \mathrm{d} \xi \mathrm{d} s .
$$

Thus, $I_{\tilde{t}}(T-\tilde{t}) \equiv 0$ implies that $\operatorname{var}\left(I_{\tilde{t}}(T-\tilde{t})\right) \equiv 0$. Hence, we can deduce that

$$
h(T-t, t ; \xi, s)=h(T-\tilde{t}, \tilde{t} ; \xi, s)
$$

for almost every $\xi$ and $s$. Finally, we can conclude that there exists a null set $N \in \mathbb{R}^{2}$ such that $t \mapsto h(T-t, t ; \xi, s)$ is a constant for all $(\xi, s) \notin N$.

Remark A.1. We see that the martingale condition for general ambit fields essentially suggests a model structure like the one we have chosen in this paper if one wants to model by a martingale. 


\section{Acknowledgements}

We would like to thank the editor and two anonymous referees for very valuable and constructive comments on an earlier draft of this article. Also, O. E. Barndorff-Nielsen appreciates support from CREATES (DNRF78), funded by the Danish National Research Foundation, and from the T. N. Thiele Centre for Applied Mathematics in Natural Science at Aarhus University. F. E. Benth acknowledges financial support from the Norwegian Research Council through the project 'Energy markets: modelling, optimization and simulation' (EMMOS), eVita 205328. A. E. D. Veraart acknowledges financial support by CREATES (DNRF78), funded by the Danish National Research Foundation, and by a Marie Curie FP7 Integration Grant within the 7th European Union Framework Programme.

\section{References}

[1] Albeverio, S., Lytvynov, E. And Mahnig, A. (2004). A model of the term structure of interest rates based on Lévy fields. Stoch. Process. Appl. 114, 251-263.

[2] Andresen, A., Koekebakker, S. and Westgaard, S. (2010). Modeling electricity forward prices using the multivariate normal inverse Gaussian distribution. J. Energy Markets 3, 1-23.

[3] Audet, N., Heiskanen, P., Keppo, J. And Vehviläinen, I. (2004). Modelling electricity forward curve dynamics in the Nordic market. In Modelling Prices in Competitive Electricity Markets, ed. D. W. Bunn, John Wiley, Chichester, pp. 251-266.

[4] Barlow, M. T. (2002). A diffusion model for electricity prices. Math. Finance 12, 287-298.

[5] Barlow, M., Gusev, Y. AND LaI, M. (2004). Calibration of multifactor models in electricity markets. Internat. J. Theoret. Appl. Finance 7, 101-120.

[6] Barndorff-Nielsen, O. E. AND Schmiegel, J. (2004). Lévy-based spatial-temporal modelling, with applications to turbulence. Uspekhi Mat. NAUK 59, 63-90.

[7] Barndorff-Nielsen, O. E. ANd Schmiegel, J. (2007). Ambit processes: with applications to turbulence and tumour growth. In Stochastic Analysis and Applications (Abel Symp. 2), Springer, Berlin, pp. 93-124.

[8] Barndorff-Nielsen, O. E. And Schmiegel, J. (2008). A stochastic differential equation framework for the timewise dynamics of turbulent velocities. Theory Prob. Appl. 52, 372-388.

[9] Barndorff-Nielsen, O. E. And Schmiegel, J. (2008). Time change and universality in turbulence. Res. Rep. 2007-8, Thiele Centre for Applied Mathematics in Natural Science, Aarhus University.

[10] Barndorff-Nielsen, O. E. And Schmiegel, J. (2008). Time change, volatility, and turbulence. In Mathematical Control Theory and Finance, Springer, Berlin, pp. 29-53.

[11] Barndorff-Nielsen, O. E. ANd Schmiegel, J. (2009). Brownian semistationary processes and volatility/intermittency. In Advanced Financial Modelling (Radon Ser. Comput. Appl. Math. 8). De Gruyter, Berlin, pp. 1-25.

[12] Barndorff-Nielsen, O. E. And Shephard, N. (2001). Non-Gaussian Ornstein-Uhlenbeck-based models and some of their uses in financial economics. J. R. Statist. Soc. B 63, 167-241.

[13] Barndorff-Nielsen, O. E. And Shiryaev, A. (2010). Change of Time and Change of Measure (Adv. Ser. Statist. Sci. Appl. Prob. 13). World Scientific, Hackensack, NJ.

[14] Barndorff-Nielsen, O. E., Benth, F. E. and Veraart, A. E. D. (2011). Ambit processes and stochastic partial differential equations. In Advanced Mathematical Methods for Finance, Springer, Heidelberg, pp. 35-74.

[15] Barndorff-Nielsen, O. E., Benth, F. E. and Veraart, A. E. D. (2014). Recent advances in ambit stochastics with a view towards tempo-spatial stochastic volatility/intermittency. Banach Center Publications. To appear.

[16] Barndorff-Nielsen, O. E., Benth, F. E. and Veraart, A. E. D. (2013). Modelling energy spot prices by volatility modulated Lévy-driven Volterra processes. Bernoulli 19, 803-845.

[17] Barth, A. And Benth, F. E. (2014). The forward dynamics in energy markets - infinite-dimensional modelling and simulation. To appear in Stochastics.

[18] Benth, F. E. (2011). The stochastic volatility model of Barndorff-Nielsen and Shephard in commodity markets. Math. Finance 21, 595-625.

[19] Benth, F. E. And Koekebakker, S. (2008). Stochastic modelling of financial electricity contracts. Energy Economics 30, 1116-1157.

[20] Benth, F. E., Cartea, A. And Kiesel, R. (2008). Pricing forward contracts in power markets by the certainty equivalence principle: explaining the sign of the market risk premium. J. Banking Finance 32, 2006-2021.

[21] Benth, F. E., Kallsen, J. And Meyer-Brandis, T. (2007). A non-Gaussian Ornstein-Uhlenbeck process for electricity spot price modeling and derivatives pricing. Appl. Math. Finance 14, 153-169. 
[22] Benth, F. E., Šaltytė Benth, J. and Koekebakker, S. (2008). Stochastic Modelling of Electricity and Related Markets (Adv. Ser. Statist. Sci. Appl. Prob. 11). World Scientific, Hackensack, NJ.

[23] Bernhardt, C., Klüppelberg, C. and Meyer-Brandis, T. (2008). Estimating high quantiles for electricity prices by stable linear models. J. Energy Markets 1, 3-19.

[24] Bichteler, K. (2002). Stochastic Integration with Jumps (Encyclopedia Math. Appl. 89). Cambridge University Press.

[25] Bjerksund, P., Rasmussen, H. and Stensland, G. (2010). Valuation and risk management in the Norwegian electricity market. In Energy, Natural Resources and Environmental Economics, eds E. Bjørndal, et al. Springer, Berlin, pp. 167-185.

[26] Brockwell, P. J. (2001). Continuous-time ARMA processes. In Stochastic Processes: Theory and Methods (Handbook Statist. 19), North-Holland, Amsterdam, pp. 249-276.

[27] Brockwell, P. J. (2001). Lévy-driven CARMA processes. Ann. Ins. Statist. Math. 53, 113-124.

[28] Carmona, R. A. and Tehranchi, M. R. (2006). Interest Rate Models: An Infinite Dimensional Stochastic Analysis Perspective. Springer, Berlin.

[29] Carr, P. And Madan, D. (1998). Option valuation using the fast Fourier transform. J. Computational Finance 2, 61-73.

[30] Cartea, A. and Figueroa, M. G. (2005). Pricing in electricity markets: a mean reverting jump diffusion model with seasonality. Appl. Math. Finance 12, 313-335.

[31] Chung, K. L. (2001). A Course in Probability Theory, 3rd edn. Academic Press, San Diego, CA.

[32] Clewlow, L. and Strickland, C. (2000). Energy Derivatives: Pricing and Risk Management. Lacima, Houston, TX.

[33] Da Prato, G. And ZabczyK, J. (1992). Stochastic Equations in Infinite Dimensions. Cambridge University Press.

[34] Delbaen, F. and Schachermayer, W. (1994). A general version of the fundamental theorem of asset pricing. Math. Ann. 300, 463-520.

[35] Diko, P., Lawford, S. And Limpens, V. (2006). Risk premia in electricity forward prices. Studies Nonlinear Dynamics Econometrics 10, Article 7.

[36] Eydeland, A. And Wolyniec, K. (2003). Energy and Power Risk Management. John Wiley, Hoboken, NJ.

[37] Folland, G. B. (1984). Real Analysis. Modern Techniques and their Applications. John Wiley, New York.

[38] Frestad, D., Benth, F. E. And Koekebakker, S. (2010). Modeling term structure dynamics in the Nordic electricity swap market. Energy J. 31, 53-86.

[39] García, I., KlÜPpelberg, C. And MÜller, G. (2011). Estimation of stable CARMA models with an application to electricity spot prices. Statist. Modelling 11, 447-470.

[40] Geman, H. (2005). Commodities and Commodity Derivatives. John Wiley, Chichester.

[41] Geman, H. and Roncoroni, A. (2006). Understanding the fine structure of electricity prices. J. Business 79, $1225-1261$.

[42] Geman, H. And VASiceK, O. (2001). Forwards and futures on non-storable commodities: the case of electricity. RISK, August, 2001.

[43] Goldstein, R. S. (2000). The term structure of interest rates as a random field. Rev. Financial Studies 13, 365-384.

[44] Hambly, B., Howison, S. and Kluge, T. (2009). Modelling spikes and pricing swing options in electricity markets. Quant. Finance 9, 937-949.

[45] Heath, D., Jarrow, R. and Morton, A. (1992). Bond pricing and the term structure of interest rates: a new methodology for contingent claims valuation. Econometrica 60, 77-105.

[46] Hikspoors, S. and Jaimungal, S. (2008). Asymptotic pricing of commodity derivatives using stochastic volatility spot models. Appl. Math. Finance 15, 449-477.

[47] Hinz, J., von Grafenstein, L., Verschuere, M. and Wilhelm, M. (2005). Pricing electricity risk by interest rate methods. Quant. Finance 5, 49-60.

[48] Kallenberg, O. (2002). Foundations of Modern Probability, 2nd edn. Springer, New York.

[49] Kallsen, J. And Shiryaev, A. N. (2002). The cumulant process and Esscher's change of measure. Finance Stoch. 6, 397-428.

[50] Kennedy, D. P. (1994). The term structure of interest rates as a Gaussian random field. Math. Finance 4, 247-258.

[51] Kennedy, D. P. (1997). Characterizing Gaussian models of the term structure of interest rates. Math. Finance 7, 107-118.

[52] Kiesel, R., Schindlmayr, G. And Börger, R. H. (2009). A two-factor model for the electricity forward market. Quant. Finance 9, 279-287.

[53] Kimmel, R. L. (2004). Modeling the term structure of interest rates: a new approach. J. Financial Econom. 72, 143-183. 
[54] Koekebakker, S. and Ollmar, F. (2005). Forward curve dynamics in the Nordic electricity market. Managerial Finance 31, 73-94.

[55] Lucia, J. J. AND Schwartz, E. S. (2002). Electricity prices and power derivatives: evidence from the Nordic power exchange. Rev. Derivatives Res. 5, 5-50.

[56] Rajput, B. S. AND Rosiński, J. (1989). Spectral representations of infinitely divisible processes. Prob. Theory Relat. Fields 82, 451-487.

[57] Samorodnitsky, G. and Taqqu, M. S. (1994). Stable Non-Gaussian Random Processes. Stochastic Models with Infinite Variance. Chapman \& Hall, New York.

[58] Samuelson, P. (1965). Proof that properly anticipated prices fluctuate randomly. Industrial Manag. Rev. 6, 41-49.

[59] Santa-Clara, P. and Sornette, D. (2001). The dynamics of the forward interest rate curve with stochastic string shocks. Rev. Financial Studies 14, 149-185.

[60] Sato, K.-I. (1999). Lévy Processes and Infinitely Divisible Distributions. Cambridge University Press.

[61] SATo, K.-I. (2004). Stochastic integrals in additive processes and application to semi-Lévy processes. Osaka J. Math. 41, 211-236.

[62] Shiryaev, A. N. (1999). Essentials of Stochastic Finance. Facts, Models, Theory (Adv. Ser. Statist. Sci. Appl. Prob. 3). World Scientific, River Edge, NJ.

[63] Vedel Jensen, E. B., Jónsdóttir, K. Y., Schmiegel, J. and Barndorff-Nielsen, O. E. (2006). Spatiotemporal modelling - with a view to biological growth. In Statistical Methods for Spatio-Temporal Systems, eds B. Finkenstädt et al., Chapman \& Hall/CRC, Boca Raton, FL, pp. 47-76.

[64] Veraart, A. E. D. and Veraart, L. A. M. (2013). Risk premiums in energy markets. J. Energy Markets 6, 1-42.

[65] Veraart, A. E. D. And Veraart, L. A. M. (2014). Modelling electricity day-ahead prices by multivariate Lévy semistationary processes. In Quantitative Energy Finance, eds F. E. Benth et al., Springer, New York, pp. 157-188.

[66] Walsh, J. B. (1986). An introduction to stochastic partial differential equations. In Ecole d'Été de Probabilités de Saint-Flour, XIV-1984 (Lecture Notes Math. 1180), Springer, Berlin, pp. 265-439.

[67] Weron, R. (2006). Modeling and Forecasting Electricity Loads and Prices. A Statistical Approach. John Wiley, Chichester. 